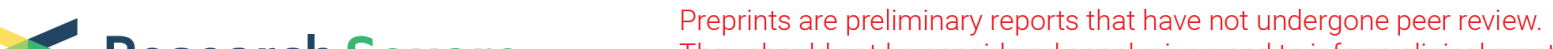 Research Square They should not be considered conclusive, used to inform clinical practice, or referenced by the media as validated information.
}

\section{Synthesis And Biological Activity of Amide Derivatives Derived From Natural Product Waltherione $\mathrm{F}$}

Hongbin Fang

Liaocheng University

Zhanfang Chen

Liaocheng University

Xuewen Hua ( $\square$ huaxuewen906@163.com )

Liaocheng University https://orcid.org/0000-0002-5748-6473

Wenrui Liu

Liaocheng University

Chenmeng Xue

Liaocheng University

Yi Liu

Liaocheng University

Xiaohe Zhu

Liaocheng University

Man Yuan

Liaocheng University

Shuang Cheng

Liaocheng University

Bingxiang Wang

Liaocheng University

Jing Ru

Liaocheng University

Dzmitry Bazhanau

Liaocheng University

Yanhong Cui

Zhejiang University of Technology

\section{Research Article}

Keywords: Waltherione F, amide compounds, structural optimization, biological activity, fluorescence characteristic 
Posted Date: November 23rd, 2021

DOI: https://doi.org/10.21203/rs.3.rs-1071215/v1

License: (9) This work is licensed under a Creative Commons Attribution 4.0 International License. Read Full License

Version of Record: A version of this preprint was published at Medicinal Chemistry Research on February 7th, 2022. See the published version at https://doi.org/10.1007/s00044-022-02852-8. 


\section{Abstract}

Structural optimization based on natural products has become an effective way to develop new green fungicides, which provide important guiding significance for practicing the new development concept and promoting the green development of pesticides. In this project, combined with the fungicidal amide lead compound X-I-4 discovered in our previous work and fungicidal piperazine derivatives reported in literatures, the target compounds containing 4-quinolone and piperazine substructures based on waltherione $\mathrm{F}$ were designed, synthesized and screened for their biological activity. The bioassay results indicated that compounds I-3, I-5, II-3, II-7, II-10, II-11 and II-13 displayed higher inhibition rates against Rhizoctonia solani than other tested compounds. The in vitro cellular cytotoxicity assay revealed the compounds II-6 and II-11 exhibited higher cytotoxicity against HepG2 than other tested compounds. The fluorescence characteristics investigation showed that the absolute fluorescence QY value of the methanol solution of the compound I-6 was higher than those of I-2, I-3, I-7 and I-8, which was further elucidated by TD-DFT.

\section{Introduction}

Agrochemicals are important production materials for agricultural production, and provide significant material support for modern agricultural development. Fungicides are an important part of agrochemicals, which are related to the safety of national food, vegetables, and fruits, and have occupied nearly $30 \%$ of the agrochemical market. However, with the long-term and large-scale use of traditional chemical synthetic fungicides, negative problems such as environmental pollution, pathogen resistance, and poisoning to beneficial insects and microorganisms have become increasingly prominent. ${ }^{[1,2]}$ Therefore, the development of efficient, safe, low residual, and environmentally friendly green fungicides has become an inevitable trend for pesticide innovation. ${ }^{[3,4]}$

Natural products have received extensive attention from scientific researchers due to their novel structures, unique mechanism of action, wide sources, no cross-resistance, fast biodegradation, and ecofriendliness. For example, osthol, matrine, berberine, and eugenol have been successfully used in the control of agricultural pathogens. ${ }^{[5,6]}$ However, low extraction rate, difficult chemical synthesis, poor environmental stability, and poor biological activity are the problems that natural products face at present. ${ }^{[7]}$ Developing new green fungicides, which was derived from the structural optimization of natural products, can effectively deal with the problems such as low safety and high pathogen resistance of traditional fungicides, and narrow fungicidal spectrum and short duration of natural products. ${ }^{[7-11]}$

Waltherione $\mathrm{F}$ is a 4-quinolone alkaloid, which was isolated from Waltheria indica $\mathrm{L}$. of the Sterculiaceae family and found to exhibit good antifungal activity. ${ }^{[12-14]}$ In our previous work, 4-quinolone derivatives QD and quinoline derivatives $\mathbf{X}-$ II derived from natural product waltherione $\mathrm{F}$ were synthesized and evaluated for their biological activity, of which compounds QD-1 and X-II-5 were discovered to have a good fungicidal activity (Fig. 1). ${ }^{[15]}$ In this project, combined with the fungicidal amide lead compound h-I9 discovered in our previous work and fungicidal piperazine compounds P-1 and P-2 reported in 
literatures, ${ }^{[16-21]}$ the amide derivatives containing 4-quinolone and piperazine substructures were designed, prepared and screened for their biological activity (Fig. 2).

\section{Results And Discussion 2.1 Organic synthesis}

The corresponding intermediates and amide derivatives I-1 - I-9, II-1 - II-13 containing 4-quinolone group were synthesized in accordance with the procedures displayed in Scheme 1. Firstly, the material 2methoxy-5-methylaniline was reacted with dimethyl acetylene to prepare the molecule 2 , which was cyclized to give ester 3 by using polyphosphoric acid (PPA) as the condensing reagent to perform the Conrad-Limpach reaction. The carboxylic acid $\mathbf{4}$ was obtained by hydrolyzing the ester group with lithium hydroxide. Subsequently, the target molecules 1-1 - 1-9 were synthesized by the condensation reaction between the carboxylic acid 4 and substituted amines with HATU/DIEA as the condensing agent. In the preparation of the target molecules II-1 - II-13, the intermediate 5 was produced by the condensation reaction of the carboxylic acid $\mathbf{4}$ and tert-butyl piperazine-1-carboxylate with TBTU/DIEA as the condensing agent, and then further deprotected with trifluoroacetic acid to provide the amide derivative 6 . Finally, the substituted carboxylic acid was reacted, respectively, with intermediate 6 to gain the target molecules II-1 - II-13 using EDCI/HOBt as the condensing reagent. Subsequently, the obtained structures were characterized with ${ }^{1} \mathrm{H} \mathrm{NMR},{ }^{13} \mathrm{C}$ NMR and HRMS. In view of the melting points of the target molecules, and combined with the TLC monitoring results, the target molecules exhibited the characteristics of strong polarity. In addition, the crystal of compound I-6 was cultivated from methanol and DCM, and determined on a Bruker D8 Venture diffractometer to provide several structural characteristics (Fig. 3, CCDC Number 2112787).

\subsection{Fungicidal activity and in vitro cellular cytotoxicity}

The in vitro inhibitory activities of the target molecules against the common agricultural pathogens was investigated, and the results are shown in Table 1. From the data, the compounds I-1 - I-9 and II-1 - II-13 showed weak fungicidal activity against the tested agricultural pathogens, which may be related to the strong polarity of the compounds. However, in a given category, several compounds displayed higher inhibition rates against the specific tested pathogens than other compounds. For example, the compounds I-3, I-5, II-3, II-7, II-11 and II-13 exhibited better fungicidal activity against Rhizoctonia solani than other compounds. Moreover, the compounds I-8 and II-6 showed better fungicidal activity against Colletotrichum capsicithan other compounds. From the perspective of structural characteristics, there was no significant difference in fungicidal activity between $\mathbf{I - 1}-\mathbf{I - 9}$ and II-1 - II-13. Considering the antitumor activity of waltherione $\mathrm{F}$ reported in literatures, the in vitro cellular cytotoxicity assay of the target compounds against HepG2 at $100 \mu \mathrm{M}$ was performed, and the results was shown in Table2. From the data, it could be observed that the compounds I-1 - I-9 and II-1 - II-13 showed weak antitumor activity 
against HepG2. However, the compounds II-6 and II-11 exhibited higher cytotoxicity than other tested compounds. 
Table 1

The in vitro inhibitory activity of the target compounds at $100 \mu \mathrm{mol} / \mathrm{L}$

\begin{tabular}{|c|c|c|c|c|c|c|c|c|}
\hline \multirow[t]{2}{*}{ Compounds } & \multicolumn{8}{|c|}{ Inhibition rate/\% } \\
\hline & GZ & RS & CS & AK & PP & $\mathrm{BC}$ & $\mathrm{CC}$ & AS \\
\hline $1-1$ & $\begin{array}{l}7.9 \pm \\
0.62\end{array}$ & $\begin{array}{l}1.3 \pm \\
0.45\end{array}$ & $\begin{array}{l}21.5 \pm \\
0.61\end{array}$ & $\begin{array}{l}1.8 \pm \\
0.23\end{array}$ & $\begin{array}{l}3.7 \pm \\
0.34\end{array}$ & $\begin{array}{l}5.5 \pm \\
0.34\end{array}$ & $\begin{array}{l}0.6 \\
\pm 0.44\end{array}$ & $\begin{array}{l}9.5 \pm \\
0.61\end{array}$ \\
\hline $1-2$ & $\begin{array}{l}1.9 \pm \\
0.47\end{array}$ & $\begin{array}{l}0.2 \pm \\
0.52\end{array}$ & $\begin{array}{l}17.2 \pm \\
0.57\end{array}$ & $\begin{array}{l}7.9 \pm \\
0.27\end{array}$ & $\begin{array}{l}2.2 \pm \\
0.24\end{array}$ & $\begin{array}{l}11.9 \pm \\
0.36\end{array}$ & $\begin{array}{l}1.0 \pm \\
0.38\end{array}$ & $\begin{array}{l}9.9 \pm \\
0.64\end{array}$ \\
\hline $1-3$ & $\begin{array}{l}7.8 \pm \\
0.33\end{array}$ & $\begin{array}{l}38.0 \pm \\
0.22\end{array}$ & $\begin{array}{l}24.1 \pm \\
0.59\end{array}$ & $\begin{array}{l}16.6 \pm \\
0.27\end{array}$ & $\begin{array}{l}8.0 \pm \\
0.25\end{array}$ & $\begin{array}{l}5.4 \pm \\
0.31\end{array}$ & $\begin{array}{l}2.7 \pm \\
0.67\end{array}$ & $\begin{array}{l}13.9 \pm \\
0.75\end{array}$ \\
\hline $1-4$ & $\begin{array}{l}11.5 \pm \\
0.52\end{array}$ & $\begin{array}{l}0.8 \pm \\
0.52\end{array}$ & $\begin{array}{l}24.7 \pm \\
0.78\end{array}$ & $\begin{array}{l}21.8 \pm \\
0.23\end{array}$ & $\begin{array}{l}7.1 \pm \\
0.34\end{array}$ & $\begin{array}{l}14.8 \pm \\
0.36\end{array}$ & $\begin{array}{l}19.5 \pm \\
0.33\end{array}$ & $\begin{array}{l}13.2 \pm \\
0.71\end{array}$ \\
\hline $1-5$ & $\begin{array}{l}7.9 \pm \\
0.40\end{array}$ & $\begin{array}{l}44.0 \pm \\
0.33\end{array}$ & $\begin{array}{l}16.7 \pm \\
0.61\end{array}$ & $\begin{array}{l}12.8 \pm \\
0.27\end{array}$ & $\begin{array}{l}15.1 \pm \\
0.20\end{array}$ & $\begin{array}{l}12.1 \pm \\
0.49\end{array}$ & $\begin{array}{l}26.3 \pm \\
0.38\end{array}$ & $\begin{array}{l}13.8 \pm \\
0.33\end{array}$ \\
\hline $1-6$ & $\begin{array}{l}14.4 \pm \\
0.33\end{array}$ & $\begin{array}{l}26.1 \pm \\
0.43\end{array}$ & $\begin{array}{l}12.6 \pm \\
0.57\end{array}$ & $\begin{array}{l}13.3 \pm \\
0.28\end{array}$ & $\begin{array}{l}12.2 \pm \\
0.20\end{array}$ & $\begin{array}{l}3.3 \pm \\
0.43\end{array}$ & $\begin{array}{l}26.5 \pm \\
0.46\end{array}$ & $\begin{array}{l}8.6 \pm \\
0.43\end{array}$ \\
\hline $1-7$ & $\begin{array}{l}11.3 \pm \\
0.33\end{array}$ & $\begin{array}{l}2.2 \pm \\
0.32\end{array}$ & $\begin{array}{l}17.3 \pm \\
0.50\end{array}$ & $\begin{array}{l}7.3 \pm \\
0.16\end{array}$ & $\begin{array}{l}1.9 \pm \\
0.23\end{array}$ & $\begin{array}{l}14.9 \pm \\
0.29\end{array}$ & $\begin{array}{l}0.9 \pm \\
0.44\end{array}$ & $\begin{array}{l}11.5 \pm \\
0.59\end{array}$ \\
\hline $1-8$ & $\begin{array}{l}14.1 \pm \\
0.59\end{array}$ & $\begin{array}{l}1.1 \pm \\
0.59\end{array}$ & $\begin{array}{l}21.1 \pm \\
049\end{array}$ & $\begin{array}{l}14.5 \pm \\
0.28\end{array}$ & $\begin{array}{l}1.2 \pm \\
0.17\end{array}$ & $\begin{array}{l}6.8 \pm \\
0.27\end{array}$ & $\begin{array}{l}37.4 \pm \\
0.36\end{array}$ & $\begin{array}{l}19.3 \pm \\
0.77\end{array}$ \\
\hline $1-9$ & $\begin{array}{l}11.2 \pm \\
0.62\end{array}$ & $\begin{array}{l}1.1 \pm \\
0.33\end{array}$ & $\begin{array}{l}20.1 \pm \\
0.78\end{array}$ & $\begin{array}{l}7.0 \pm \\
0.32\end{array}$ & $\begin{array}{l}7.4 \pm \\
0.29\end{array}$ & $\begin{array}{l}19.7 \pm \\
0.73\end{array}$ & $\begin{array}{l}1.2 \pm \\
0.44\end{array}$ & $\begin{array}{l}17.3 \pm \\
0.61\end{array}$ \\
\hline 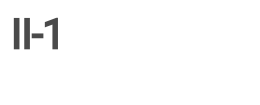 & $\begin{array}{l}17.4 \pm \\
0.55\end{array}$ & $\begin{array}{l}1.1 \pm \\
0.48\end{array}$ & $\begin{array}{l}16.9 \pm \\
0.43\end{array}$ & $\begin{array}{l}3.3 \pm \\
0.41\end{array}$ & $\begin{array}{l}5.8 \pm \\
0.45\end{array}$ & $\begin{array}{l}11.6 \pm \\
0.29\end{array}$ & $\begin{array}{l}1.0 \pm \\
0.46\end{array}$ & $\begin{array}{l}10.3 \pm \\
0.75\end{array}$ \\
\hline$\| 1-2$ & $\begin{array}{l}18.7 \pm \\
0.84\end{array}$ & $\begin{array}{l}0.9 \pm \\
0.47\end{array}$ & $\begin{array}{l}25.0 \pm \\
0.61\end{array}$ & $\begin{array}{l}14.2 \pm \\
0.27\end{array}$ & $\begin{array}{l}13.8 \pm \\
0.23\end{array}$ & $\begin{array}{l}12.8 \pm \\
0.36\end{array}$ & $\begin{array}{l}27.0 \pm \\
0.38\end{array}$ & $\begin{array}{l}5.3 \pm \\
0.51\end{array}$ \\
\hline II-3 & $\begin{array}{l}12.0 \pm \\
0.55\end{array}$ & $\begin{array}{l}37.5 \pm \\
0.45\end{array}$ & $\begin{array}{l}17.2 \pm \\
0.68\end{array}$ & $\begin{array}{l}18.0 \pm \\
0.35\end{array}$ & $\begin{array}{l}29.4 \pm \\
0.30\end{array}$ & $\begin{array}{l}2.7 \pm \\
0.48\end{array}$ & $\begin{array}{l}16.1 \pm \\
0.46\end{array}$ & $\begin{array}{l}5.7 \pm \\
0.44\end{array}$ \\
\hline II-4 & $\begin{array}{l}19.4 \pm \\
0.29\end{array}$ & $\begin{array}{l}0.7 \pm \\
0.28\end{array}$ & $\begin{array}{l}16.9 \pm \\
0.57\end{array}$ & $\begin{array}{l}22.4 \pm \\
0.20\end{array}$ & $\begin{array}{l}10.3 \pm \\
0.20\end{array}$ & $\begin{array}{l}8.8 \pm \\
0.42\end{array}$ & $\begin{array}{l}0.7 \pm \\
0.32\end{array}$ & $\begin{array}{l}12.0 \pm \\
0.52\end{array}$ \\
\hline II-5 & $\begin{array}{l}8.3 \pm \\
0.72\end{array}$ & $\begin{array}{l}12.5 \pm \\
0.33\end{array}$ & $\begin{array}{l}17.9 \pm \\
0.81\end{array}$ & $\begin{array}{l}13.6 \\
\pm 0.22\end{array}$ & $\begin{array}{l}9.8 \pm \\
0.28\end{array}$ & $\begin{array}{l}8.4 \pm \\
0.61\end{array}$ & $\begin{array}{l}23.8 \pm \\
0.43\end{array}$ & $\begin{array}{l}4.9 \pm \\
0.15\end{array}$ \\
\hline II-6 & $\begin{array}{l}16.2 \pm \\
0.83\end{array}$ & $\begin{array}{l}0.6 \pm \\
0.45\end{array}$ & $\begin{array}{l}26.0 \pm \\
0.68\end{array}$ & $\begin{array}{l}24.2 \pm \\
0.24\end{array}$ & $\begin{array}{l}15.3 \pm \\
0.20\end{array}$ & $\begin{array}{l}14.5 \pm \\
043\end{array}$ & $\begin{array}{l}37.1 \pm \\
0.38\end{array}$ & $\begin{array}{l}9.2 \pm \\
0.52\end{array}$ \\
\hline II-7 & $\begin{array}{l}14.6 \pm \\
0.28\end{array}$ & $\begin{array}{l}37.7 \pm \\
0.38\end{array}$ & $\begin{array}{l}17.2 \pm \\
0.57\end{array}$ & $\begin{array}{l}10.9 \pm \\
0.28\end{array}$ & $\begin{array}{l}11.6 \pm \\
0.34\end{array}$ & $\begin{array}{l}3.3 \\
\pm 0.36\end{array}$ & $\begin{array}{l}21.9 \pm \\
0.38\end{array}$ & $\begin{array}{l}12.9 \pm \\
0.25\end{array}$ \\
\hline II-8 & $\begin{array}{l}27.2 \pm \\
0.37\end{array}$ & $\begin{array}{l}1.7 \pm \\
0.32\end{array}$ & $\begin{array}{l}13.9 \pm \\
0.29\end{array}$ & $\begin{array}{l}2.4 \pm \\
0.19\end{array}$ & $\begin{array}{l}31.2 \pm \\
0.32\end{array}$ & $\begin{array}{l}11.9 \pm \\
0.31\end{array}$ & $\begin{array}{l}2.4 \pm \\
0.77\end{array}$ & $\begin{array}{l}5.6 \pm \\
0.58\end{array}$ \\
\hline
\end{tabular}




\begin{tabular}{|c|c|c|c|c|c|c|c|c|}
\hline \multirow[t]{2}{*}{ Compounds } & \multicolumn{8}{|c|}{ Inhibition rate/\% } \\
\hline & GZ & RS & CS & AK & PP & $\mathrm{BC}$ & $\mathrm{CC}$ & AS \\
\hline II-9 & $\begin{array}{l}11.5 \pm \\
0.59\end{array}$ & $\begin{array}{l}0.6 \pm \\
0.37\end{array}$ & $\begin{array}{l}12.9 \pm \\
0.74\end{array}$ & $\begin{array}{l}4.9 \pm \\
0.27\end{array}$ & $\begin{array}{l}2.0 \pm \\
0.19\end{array}$ & $\begin{array}{l}8.3 \pm \\
0.40\end{array}$ & $\begin{array}{l}2.5 \pm \\
0.56\end{array}$ & $\begin{array}{l}4.5 \pm \\
0.45\end{array}$ \\
\hline II-10 & $\begin{array}{l}4.8 \pm \\
0.37\end{array}$ & $\begin{array}{l}35.2 \pm \\
0.40\end{array}$ & $\begin{array}{l}16.7 \pm \\
0.57\end{array}$ & $\begin{array}{l}9.2 \pm \\
0.22\end{array}$ & $\begin{array}{l}7.7 \pm \\
0.34\end{array}$ & $\begin{array}{l}8.6 \pm \\
0.25\end{array}$ & $\begin{array}{l}30.4 \pm \\
0.38\end{array}$ & $\begin{array}{l}9.8 \pm \\
0.59\end{array}$ \\
\hline II-11 & $\begin{array}{l}10.5 \pm \\
0.45\end{array}$ & $\begin{array}{l}38.3 \pm \\
0.33\end{array}$ & $\begin{array}{l}16.4 \pm \\
0.44\end{array}$ & $\begin{array}{l}15.0 \pm \\
0.20\end{array}$ & $\begin{array}{l}2.3 \pm \\
0.28\end{array}$ & $\begin{array}{l}3.3 \pm \\
0.17\end{array}$ & $\begin{array}{l}1.6 \pm \\
0.68\end{array}$ & $\begin{array}{l}9.5 \pm \\
0.66\end{array}$ \\
\hline $\mathrm{II}-12$ & $\begin{array}{l}20.8 \pm \\
0.48\end{array}$ & $\begin{array}{l}1.0 \pm \\
0.47\end{array}$ & $\begin{array}{l}20.3 \pm \\
0.52\end{array}$ & $\begin{array}{l}6.6 \pm \\
0.27\end{array}$ & $\begin{array}{l}1.4 \pm \\
0.23\end{array}$ & $\begin{array}{l}14.7 \pm \\
0.43\end{array}$ & $\begin{array}{l}0.6 \pm \\
0.52\end{array}$ & $\begin{array}{l}12.8 \pm \\
0.45\end{array}$ \\
\hline II-13 & $\begin{array}{l}11.6 \pm \\
0.33\end{array}$ & $\begin{array}{l}36.9 \pm \\
0.37\end{array}$ & $\begin{array}{l}21.1 \pm \\
0.61\end{array}$ & $\begin{array}{l}15.3 \pm \\
0.18\end{array}$ & $\begin{array}{l}16.0 \pm \\
0.36\end{array}$ & $\begin{array}{l}20.5 \pm \\
0.30\end{array}$ & $\begin{array}{l}21.5 \pm \\
0.44\end{array}$ & $\begin{array}{l}11.8 \pm \\
0.52\end{array}$ \\
\hline Fluopyram & 100 & $\begin{array}{l}61.5 \pm \\
0.38\end{array}$ & 100 & $\begin{array}{l}94.5 \pm \\
0.23\end{array}$ & $\begin{array}{l}31.8 \pm \\
0.16\end{array}$ & $\begin{array}{l}60.7 \pm \\
0.48\end{array}$ & $\begin{array}{l}51.8 \pm \\
0.32\end{array}$ & 100 \\
\hline Carbendazim & 100 & 100 & $\begin{array}{l}12.1 \pm \\
0.49\end{array}$ & $\begin{array}{l}13.3 \pm \\
0.20\end{array}$ & 100 & $\begin{array}{l}90.1 \pm \\
0.21\end{array}$ & 100 & $\begin{array}{l}7.3 \pm \\
0.51\end{array}$ \\
\hline
\end{tabular}

GZ, Gibberella zeae; RS, Rhizoctonia solani; CS, Cercospora circumscissa Sacc.; AK, Alternaria kikuchiana Tanaka; PP, Physalospora piricola; BC, Botrytis cinerea; CC, Colletotrichum capsici; AS, Alternaria sp.

Table 2

Cytotoxic profiles of the target compounds against HepG2 at $100 \mu \mathrm{M}$

\begin{tabular}{|llllll|}
\hline Compounds & Cytotoxicity/\% & Compounds & Cytotoxicity/\% & Compounds & Cytotoxicity/\% \\
\hline I-1 & $5.8 \pm 0.31$ & II- & $4.5 \pm 1.13$ & II-10 & 0 \\
\hline I-2 & $1.1 \pm 1.06$ & II-2 & $12.2 \pm 0.88$ & II-11 & $25.6 \pm 2.06$ \\
\hline I-3 & $2.7 \pm 0.59$ & II-3 & $5.9 \pm 0.16$ & II-12 & $16.3 \pm 1.31$ \\
\hline I-4 & $7.9 \pm 1.67$ & II-4 & 0 & I-13 & $17.5 \pm 0.80$ \\
\hline I-5 & $6.1 \pm 1.66$ & II-5 & $6.8 \pm 1.25$ & & 100 \\
\hline I-6 & 0 & II-6 & $26.2 \pm 1.35$ & & \\
\hline H-7 & $3.3 \pm 0.41$ & II-7 & $8.8 \pm 1.14$ & & \\
\hline I-8 & 0 & II-8 & $13.9 \pm 0.94$ & & \\
\hline H-9 & $9.5 \pm 0.34$ & II-9 & 0 & & \\
\hline
\end{tabular}

\subsection{Fluorescence Characteristic Investigation}

Considering the fluorescence characteristics of 4-quinolone derivatives QD and quinoline derivatives X-II exhibited in our previous work, the UV-vis absorption and fluorescence emission spectra of the 
compounds I-1 - I-9 and II-1 - II-13 were scanned and shown in Fig. 4. From the data, the compounds I-6 and $\mathbf{~ I - 7 ~ d i s p l a y e d ~ a ~ s t r o n g e r ~ f l u o r e s c e n c e ~ i n t e n s i t y ~ t h a n ~ o t h e r ~ c o m p o u n d s . ~ A f t e r w a r d s , ~ t h e ~ a b s o l u t e ~}$ fluorescence QY values of the methanol solutions $(50 \mu \mathrm{M})$ of the compounds I-2, I-3, I-6, I-7 and I-8 were measured to further illustrate the fluorescence characteristics, and the results were exhibited in Table 3 . In could be found that compound I-6 exhibited a higher QY than that of $1-2, I-3, I-7$, and I-8, with a value of $27.4 \%$. Subsequently, the TD-DFT calculations of molecules $1-6$ and $1-7$ were performed to elucidate the obtained fluorescence properties (Fig. 5). From the data, the HOMO orbital energy and LUMO orbital energy of molecule $1-6$ were both higher than that of $1-7$, which was conducive to the energy level transitions of electrons in the HOMO orbital of molecule I-6. In the meantime, the molecule I-6 exhibited a larger energy gap than $\mathbf{1 - 7}$, which help $1-6$ absorb more energy to produce stronger fluorescence intensity.

Table 3

The absolute fluorescence quantum yields (QY) of compounds I-2, I-3, I-6, I-7 and $\mathrm{I}-8$ in methanol

\begin{tabular}{|llllll|}
\hline Compounds & QY (\%) & Compounds & QY (\%) & Compounds & QY (\%) \\
\hline I-2 & 19.6 & I-6 & 27.4 & I-8 & 11.4 \\
I-3 & 15.5 & I-7 & 15.5 & & \\
\hline
\end{tabular}

\section{Conclusion}

In summary, twenty-two novel amide derivatives derived from the structural modification of waltherione $\mathrm{F}$ were designed and synthesized. The obtained structures were characterized by ${ }^{1} \mathrm{H}$ NMR, ${ }^{13} \mathrm{C}$ NMR and HRMS. Several crystal structural characteristics were also revealed via X-ray crystal diffraction of compounds I-6. The bioassay results indicated that the compounds I-1 - I-9 and II-1 - II-13 showed weak inhibitory activities against the tested agricultural pathogens. However, in a given category, several compounds displayed higher inhibition rates against Rhizoctonia solani than other compounds. The in vitro cellular cytotoxicity assay revealed the compounds II-6 and II-11 exhibited higher cytotoxicity against HepG2 than other tested compounds. The fluorescence characteristics investigation showed that the QY value of the methanol solution of the compound I-6 was higher than that of I-2, I-3, I-7 and I-8, which was further explained by TD-DFT.

\section{Experimental}

\subsection{Material and instruments}

The materials and reagents used in the organic synthesis reactions were of analytical grade, and purchased from Energy Chemical and Bide Pharmatech Ltd. Melting points were measured on an X-5 binocular microscope. ${ }^{1} \mathrm{H}$ - and ${ }^{13} \mathrm{C}$ - NMR were provided on a Bruker-500 $\mathrm{MHz}$ spectrometer. HRMS (Waters Xevo G2-XS QTof, USA) was used to record the relative molecular mass. X-ray crystal structure 
was determined on a Bruker D8 Venture diffractometer. The purification of target compounds was performed by the column chromatography on silica gel (200-300 mesh).

\subsection{Preparation of the target molecules 4.2.1 Synthetic procedure for I-1-I-9}

8-methoxy-5-methyl-4-oxo- $N$-phenyl-1,4-dihydro-quinoline-2-carboxamide (I-1) was synthesized as follows. To a mixture of 8-methoxy-5-methyl-4-oxo-1,4-dihydroquinoline-2-carboxylic acid (0.50 g, $2.1 \mathrm{mmol})$, HATU $(1.14 \mathrm{~g}, 3.0 \mathrm{mmol})$, DIEA $(0.66 \mathrm{~g}, 5.1 \mathrm{mmol})$ in DMF $(10 \mathrm{~mL})$ was added aniline $(0.24 \mathrm{~g}, 2.6 \mathrm{mmol})$. The solution was reacted for $12 \mathrm{~h}$ at room temperature, and then poured into DCM $(100 \mathrm{~mL})$. The organic phase was washed with water $(100 \mathrm{~mL})$, and separated by extraction. The obtained organic layer was dried, filtered and concentrated. The resulting residue was purified by chromatograph on silica gel using $\mathrm{DCM} /$ methanol $(\mathrm{v} / \mathrm{v}=50 / 1)$ as eluent to obtain $\mathrm{I}-1$. Compounds $\mathrm{I}-2 \mathrm{-} \mathbf{I}-\mathbf{9}$ were prepared similarly.

8-methoxy-5-methyl-4-oxo- $N$-phenyl-1,4-dihydroquinoline-2-carboxamide (I-1): White solid, yield 56\%, m.p. 277-278 ${ }^{\circ}$ C. ${ }^{1} \mathrm{H}$ NMR (DMSO-d $\left.{ }_{6}, 500 \mathrm{MHz}\right) \delta 10.69(1 \mathrm{H}, \mathrm{s}, \mathrm{NH}), 9.83(1 \mathrm{H}, \mathrm{s}, \mathrm{CONH}), 7.81(2 \mathrm{H}, \mathrm{d}, J=7.8 \mathrm{~Hz}$, $\mathrm{Ph}-\mathrm{H}), 7.41(2 \mathrm{H}, \mathrm{t}, J=7.9 \mathrm{~Hz}, \mathrm{Ph}-\mathrm{H}), 7.23-7.16(2 \mathrm{H}, \mathrm{m}, \mathrm{Ph}-\mathrm{H}), 7.05-6.99(2 \mathrm{H}, \mathrm{m}$, quinolone-H and Ph-H), $4.00\left(3 \mathrm{H}, \mathrm{s}, \mathrm{OCH}_{3}\right), 2.73\left(3 \mathrm{H}, \mathrm{s}, \mathrm{Ph}-\mathrm{CH}_{3}\right) .{ }^{13} \mathrm{C} \mathrm{NMR}$ (DMSO-d $\left.{ }_{6}, 126 \mathrm{MHz}\right) \delta 180.8,160.5,146.9,139.0$, 138.3, 131.8, 130.2, 129.3, 125.7, 125.3, 124.4, 121.3, 111.9, 110.0, 56.9, 22.6. HRMS (ESI $\left.{ }^{+}\right) \mathrm{m} / \mathrm{z}$ calcd for $\mathrm{C}_{18} \mathrm{H}_{17} \mathrm{~N}_{2} \mathrm{O}_{3} 309.1234$, found $309.1233[\mathrm{M}+\mathrm{H}]^{+}$.

$N$-(2,3-dichlorophenyl)-8-methoxy-5-methyl-4-oxo-1,4-dihydroquinoline-2-carboxamide (1-2): White solid, yield $43 \%$, m.p. $283-284^{\circ} \mathrm{C} .{ }^{1} \mathrm{H}$ NMR $\left(\mathrm{CD}_{2} \mathrm{Cl}_{2}+\mathrm{CD}_{3} \mathrm{OD}+\mathrm{DMSO}_{6}, 500 \mathrm{MHz}\right) \delta 8.63-8.57(1 \mathrm{H}, \mathrm{m}, \mathrm{Ph}-\mathrm{H})$, $8.00-7.96(1 \mathrm{H}, \mathrm{m}, \mathrm{Ph}-\mathrm{H}), 7.62$ - $7.56(1 \mathrm{H}, \mathrm{m}, \mathrm{Ph}-\mathrm{H}), 7.44-7.36$ (2H, m, Ph-H and quinolone-H), $7.35-$

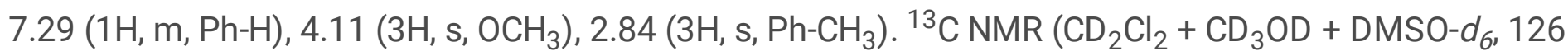
$\mathrm{MHz}) \delta 161.2,159.1,158.7,154.2,148.3,141.4,132.9,132.7,128.4,125.6,124.2,120.8,118.9,110.5$, 104.7, 56.3, 23.1. HRMS $\left(\mathrm{ESI}^{+}\right) \mathrm{m} / \mathrm{z}$ calcd for $\mathrm{C}_{18} \mathrm{H}_{15} \mathrm{Cl}_{2} \mathrm{~N}_{2} \mathrm{O}_{3} 377.0454$, found $377.0453[\mathrm{M}+\mathrm{H}]^{+}$.

8-methoxy-5-methyl-4-oxo- $N$-(2-(trifluoromethyl)phenyl)-1,4-dihydroquinoline-2-carboxamide (I-3): White solid, yield 60\%, m.p. 232-234 ${ }^{\circ} \mathrm{C} .{ }^{1} \mathrm{H}$ NMR $\left(\mathrm{CDCl}_{3}, 500 \mathrm{MHz}\right) \delta 9.79(1 \mathrm{H}, \mathrm{s}, \mathrm{NH}), 9.72(1 \mathrm{H}, \mathrm{s}, \mathrm{CONH}), 7.97$ $(1 \mathrm{H}, \mathrm{d}, J=8.1 \mathrm{~Hz}, \mathrm{Ph}-\mathrm{H}), 7.73(1 \mathrm{H}, \mathrm{d}, J=7.8 \mathrm{~Hz}, \mathrm{Ph}-\mathrm{H}), 7.66(1 \mathrm{H}, \mathrm{t}, J=7.7 \mathrm{~Hz}, \mathrm{Ph}-\mathrm{H}), 7.42(1 \mathrm{H}, \mathrm{t}, J=7.7 \mathrm{~Hz}$, Ph-H), $7.13(1 \mathrm{H}, \mathrm{d}, J=2.1 \mathrm{~Hz}, \mathrm{Ph}-\mathrm{H}), 6.92(2 \mathrm{H}, \mathrm{s}$, quinolone-H and $\mathrm{Ph}-\mathrm{H}), 3.98\left(3 \mathrm{H}, \mathrm{s}, \mathrm{OCH}_{3}\right), 2.57(3 \mathrm{H}, \mathrm{s}$, $\left.\mathrm{CH}_{3}\right) .{ }^{13} \mathrm{C} \mathrm{NMR}\left(\mathrm{CDCl}_{3}, 126 \mathrm{MHz}\right) \delta 181.8,160.9,146.5,137.7,134.1,132.9,131.9,131.5,127.4,126.8$, $126.6(q, J=5.0 \mathrm{~Hz}), 125.6,124.9,124.3(q, J=30.4 \mathrm{~Hz}), 123.7(q, J=273.9 \mathrm{~Hz}), 110.7,109.3,56.0,22.1$. HRMS $\left(\mathrm{ESI}^{+}\right) \mathrm{m} / \mathrm{z}$ calcd $\mathrm{C}_{19} \mathrm{H}_{16} \mathrm{~F}_{3} \mathrm{~N}_{2} \mathrm{O}_{3}$ for 377.1108 , found $377.1109[\mathrm{M}+\mathrm{H}]^{+}$.

8-methoxy-N-(2-methoxy-5-methylphenyl)-5-methyl-4-oxo-1,4-dihydroquinoline-2-carboxamide (1-4): White solid, yield $51 \%$, m.p. $211-212^{\circ} \mathrm{C} .{ }^{1} \mathrm{H}$ NMR $\left(\mathrm{CD}_{2} \mathrm{Cl}_{2}, 500 \mathrm{MHz}\right) \delta 9.71(1 \mathrm{H}, \mathrm{s}$, quinolone-NH), $8.87(1 \mathrm{H}, \mathrm{s}$, CONH), $8.29(1 \mathrm{H}, \mathrm{d}, J=1.8 \mathrm{~Hz}, \mathrm{Ph}-\mathrm{H}), 7.01(2 \mathrm{H}, \mathrm{s}, \mathrm{Ph}-\mathrm{H}), 6.99(1 \mathrm{H}, \mathrm{dd}, J=8.3,1.4 \mathrm{~Hz}, \mathrm{Ph}-\mathrm{H}), 6.89(1 \mathrm{H}, \mathrm{d}, J$ 
= $8.3 \mathrm{~Hz}, \mathrm{Ph}-\mathrm{H}), 6.64\left(1 \mathrm{H}, \mathrm{s}\right.$, quinolone-H), $4.04\left(3 \mathrm{H}, \mathrm{s}, \mathrm{OCH}_{3}\right), 3.95\left(3 \mathrm{H}, \mathrm{s}, \mathrm{OCH}_{3}\right), 2.83\left(3 \mathrm{H}, \mathrm{s}, \mathrm{Ph}-\mathrm{CH}_{3}\right), 2.37$ $\left(3 \mathrm{H}, \mathrm{s}, \mathrm{Ph}-\mathrm{CH}_{3}\right) .{ }^{13} \mathrm{C} \mathrm{NMR}\left(\mathrm{CD}_{2} \mathrm{Cl}_{2}, 126 \mathrm{MHz}\right) \delta 181.0,159.0,146.5,138.0,131.8,131.1,130.5,129.8$, 126.1, 125.4, 125.3, 120.5, 110.6, 110.1, 108.4, 100.9, 56.1, 55.9, 22.2, 20.7. HRMS (ESI ${ }^{+}$) m/z calcd for $\mathrm{C}_{20} \mathrm{H}_{21} \mathrm{~N}_{2} \mathrm{O}_{4} 353.1496$, found $353.1495[\mathrm{M}+\mathrm{H}]^{+}$.

$\mathrm{N}$-(3-isopropoxyphenyl)-8-methoxy-5-methyl-4-oxo-1,4-dihydroquinoline-2-carboxamide (1-5): White solid, yield $44 \%$, m.p. $252-254^{\circ} \mathrm{C} .{ }^{1} \mathrm{H}$ NMR $\left(\mathrm{CD}_{2} \mathrm{Cl}_{2}+\mathrm{CD}_{3} \mathrm{OD}, 500 \mathrm{MHz}\right) \delta 7.43(1 \mathrm{H}, \mathrm{s}, \mathrm{Ph}-\mathrm{H}), 7.30-7.29(2 \mathrm{H}, \mathrm{m}$, Ph-H), $7.10(1 \mathrm{H}, \mathrm{d}, J=8.1 \mathrm{~Hz}, \mathrm{Ph}-\mathrm{H}), 7.07(1 \mathrm{H}, \mathrm{d}, J=8.4 \mathrm{~Hz}, \mathrm{Ph}-\mathrm{H}), 6.95(1 \mathrm{H}, \mathrm{s}$, quinolone-H), $6.80-6.74$ $(1 \mathrm{H}, \mathrm{m}, \mathrm{Ph}-\mathrm{H}), 4.64-4.61(1 \mathrm{H}, \mathrm{m}, \mathrm{CH}), 4.05\left(3 \mathrm{H}, \mathrm{s}, \mathrm{OCH}_{3}\right), 2.82\left(3 \mathrm{H}, \mathrm{s}, \mathrm{Ph}_{-} \mathrm{CH}_{3}\right), 1.36(6 \mathrm{H}, \mathrm{d}, J=6.1 \mathrm{~Hz}$, $\left.\left(\mathrm{CH}_{3}\right)_{2}\right) \cdot{ }^{13} \mathrm{C} \mathrm{NMR}\left(\mathrm{CD}_{2} \mathrm{Cl}_{2}+\mathrm{CD}_{3} \mathrm{OD}, 126 \mathrm{MHz}\right) \delta 158.3,146.7,129.5,125.8,113.3,112.9,112.5,110.9$, 110.8, 108.8, 108.6, 70.1, 55.8, 21.9, 21.4. HRMS $\left(\mathrm{ESI}^{+}\right) \mathrm{m} / \mathrm{z}$ calcd for $\mathrm{C}_{21} \mathrm{H}_{23} \mathrm{~N}_{2} \mathrm{O}_{4} 367.1652$, found $367.1652[\mathrm{M}+\mathrm{H}]^{+}$.

$N$-(2,4-dimethoxybenzyl)-8-methoxy-5-methyl-4-oxo-1,4-dihydroquinoline-2-carboxamide (I-6): White solid, yield $40 \%$, m.p. $257-258^{\circ} \mathrm{C}$. ${ }^{1} \mathrm{H}$ NMR $\left(\mathrm{CD}_{2} \mathrm{Cl}_{2}+\mathrm{CD}_{3} \mathrm{OD}, 500 \mathrm{MHz}\right) \delta 7.23(1 \mathrm{H}, \mathrm{d}, J=8.3 \mathrm{~Hz}, \mathrm{Ph}-\mathrm{H}), 7.03$ $(2 \mathrm{H}, \mathrm{s}, \mathrm{Ph}-\mathrm{H}), 6.68(1 \mathrm{H}, \mathrm{s}$, quinolone-H), $6.51(1 \mathrm{H}, \mathrm{d}, J=2.3 \mathrm{~Hz}, \mathrm{Ph}-\mathrm{H}), 6.47(1 \mathrm{H}, \mathrm{dd}, J=8.3,2.3 \mathrm{~Hz}, \mathrm{Ph}-\mathrm{H})$, $4.55\left(2 \mathrm{H}, \mathrm{s}, \mathrm{CH}_{2}\right), 4.01\left(3 \mathrm{H}, \mathrm{s}, \mathrm{OCH}_{3}\right), 3.86\left(3 \mathrm{H}, \mathrm{s}, \mathrm{OCH}_{3}\right), 3.80\left(3 \mathrm{H}, \mathrm{s}, \mathrm{OCH}_{3}\right), 2.79\left(3 \mathrm{H}, \mathrm{s}, \mathrm{Ph}-\mathrm{CH}_{3}\right) .{ }^{13} \mathrm{C} \mathrm{NMR}$ $\left(\mathrm{CD}_{2} \mathrm{Cl}_{2}+\mathrm{CD}_{3} \mathrm{OD}, 126 \mathrm{MHz}\right) \delta 184.5,163.3,162.7,160.6,148.6,140.6,133.6,132.8,131.9,127.7,126.3$, 119.7, 112.8, 110.1, 106.0, 100.3, 57.9, 57.1, 57.1, 41.2, 24.0. HRMS $\left(E S I^{+}\right) \mathrm{m} / \mathrm{z}$ calcd for $\mathrm{C}_{21} \mathrm{H}_{23} \mathrm{~N}_{2} \mathrm{O}_{5}$ 383.1601 , found $383.1600[\mathrm{M}+\mathrm{H}]^{+}$.

8-methoxy-5-methyl-4-oxo- $N$-(3-(trifluoromethyl)benzyl)-1,4-dihydroquinoline-2-carboxamide (I-7): White solid, yield $41 \%$, m.p. $214-215^{\circ} \mathrm{C} .{ }^{1} \mathrm{H}$ NMR $\left(\mathrm{CD}_{2} \mathrm{Cl}_{2}+\mathrm{CD}_{3} \mathrm{OD}, 500 \mathrm{MHz}\right) \delta 8.11(1 \mathrm{H}, \mathrm{s}, \mathrm{NH}), 7.66(1 \mathrm{H}, \mathrm{s}, \mathrm{Ph}-$ H), $7.62(1 \mathrm{H}, \mathrm{d}, J=7.7 \mathrm{~Hz}, \mathrm{Ph}-\mathrm{H}), 7.58(1 \mathrm{H}, \mathrm{d}, J=7.8 \mathrm{~Hz}, \mathrm{Ph}-\mathrm{H}), 7.52(1 \mathrm{H}, \mathrm{t}, J=7.8 \mathrm{~Hz}, \mathrm{Ph}-\mathrm{H}), 7.06(2 \mathrm{H}, \mathrm{s}$, $\mathrm{Ph}-\mathrm{H}), 6.73\left(1 \mathrm{H}, \mathrm{s}\right.$, quinolone-H), $4.68\left(2 \mathrm{H}, \mathrm{s}, \mathrm{CH}_{2}\right), 4.03\left(3 \mathrm{H}, \mathrm{s}, \mathrm{OCH}_{3}\right), 2.80\left(3 \mathrm{H}, \mathrm{s}, \mathrm{Ph}_{-} \mathrm{CH}_{3}\right) .{ }^{13} \mathrm{C} \mathrm{NMR}$ $\left(\mathrm{CD}_{3} \mathrm{OD}, 126 \mathrm{MHz}\right) \delta 181.9,161.1,146.0,138.5,137.9,130.6,130.2,128.5,125.2,123.8,123.6,110.3$, 107.6, 55.4, 42.8, 21.4. HRMS (ESI ${ }^{+}$) $\mathrm{m} / \mathrm{z}$ calcd for $\mathrm{C}_{20} \mathrm{H}_{18} \mathrm{~F}_{3} \mathrm{~N}_{2} \mathrm{O}_{3} 391.1264$, found $391.1261[\mathrm{M}+\mathrm{H}]^{+}$.

8-methoxy-5-methyl-4-oxo- $N$-(3',4',5'-trifluoro-[1,1'-biphenyl]-4-yl)-1,4-dihydroquinoline-2-carboxamide (I-8): White solid, yield $39 \%$, m.p. $233-234^{\circ} \mathrm{C} .{ }^{1} \mathrm{H}$ NMR $\left(\mathrm{CD}_{2} \mathrm{Cl}_{2}, 500 \mathrm{MHz}\right) \delta 10.13(1 \mathrm{H}$, s, quinolone-NH), 9.66 $(1 \mathrm{H}, \mathrm{s}, \mathrm{CONH}), 7.67(1 \mathrm{H}, \mathrm{d}, J=7.8 \mathrm{~Hz}, \mathrm{Ph}-\mathrm{H}), 7.42(1 \mathrm{H}, \mathrm{t}, J=7.4 \mathrm{~Hz}, \mathrm{Ph}-\mathrm{H}), 7.35(1 \mathrm{H}, \mathrm{t}, J=7.5 \mathrm{~Hz}, \mathrm{Ph}-\mathrm{H})$, $7.31(1 \mathrm{H}, \mathrm{d}, J=7.4 \mathrm{~Hz}, \mathrm{Ph}-\mathrm{H}), 7.09(1 \mathrm{H}, \mathrm{s}$, quinolone-H), $7.04(2 \mathrm{H}, \mathrm{dd}, J=8.1,6.8 \mathrm{~Hz}, \mathrm{Ph}-\mathrm{H}), 6.88(1 \mathrm{H}, \mathrm{d}, J=$ $8.0 \mathrm{~Hz}, \mathrm{Ph}-\mathrm{H}), 6.84(1 \mathrm{H}, \mathrm{d}, J=8.1 \mathrm{~Hz}, \mathrm{Ph}-\mathrm{H}), 3.90\left(3 \mathrm{H}, \mathrm{s}, \mathrm{OCH}_{3}\right), 2.37\left(3 \mathrm{H}, \mathrm{s}, \mathrm{Ph}-\mathrm{CH}_{3}\right) .{ }^{13} \mathrm{C} \mathrm{NMR}\left(\mathrm{CD}_{2} \mathrm{Cl}_{2}\right.$, $126 \mathrm{MHz}) \delta 181.0,159.8,145.9,137.4,134.5,132.8,131.1,130.1,129.5,128.4,126.7,126.4,124.8,123.9$, $112.5(\mathrm{~d}, J=5.3 \mathrm{~Hz}), 112.4(\mathrm{~d}, J=4.9 \mathrm{~Hz}), 110.0,108.2,55.3,21.2$. HRMS $\left(\mathrm{ESI}^{+}\right) \mathrm{m} / \mathrm{z}$ calcd for $\mathrm{C}_{24} \mathrm{H}_{18} \mathrm{~F}_{3} \mathrm{~N}_{2} \mathrm{O}_{3} 439.1264$, found $439.1265[\mathrm{M}+\mathrm{H}]^{+}$. 
$N$-(4'-chloro-[1,1'-biphenyl]-4-yl)-8-methoxy-5-methyl-4-oxo-1,4-dihydroquinoline-2-carboxamide (1-9): White solid, yield $40 \%$, m.p. $170-171^{\circ} \mathrm{C} .{ }^{1} \mathrm{H}$ NMR $\left(\mathrm{CD}_{2} \mathrm{Cl}_{2}, 500 \mathrm{MHz}\right) \delta 10.23(1 \mathrm{H}, \mathrm{s}, \mathrm{NH}), 8.55(1 \mathrm{H}, \mathrm{dd}, J=8.2$, $0.7 \mathrm{~Hz}, \mathrm{Ph}-\mathrm{H}), 7.52(1 \mathrm{H}, \mathrm{s}, \mathrm{Ph}-\mathrm{H}), 7.47-7.44(2 \mathrm{H}, \mathrm{m}, \mathrm{Ph}-\mathrm{H}), 7.42-7.35(4 \mathrm{H}, \mathrm{m}, \mathrm{Ph}-\mathrm{H}$ and quinolone-H), $7.25(1 \mathrm{H}, \mathrm{dd}, J=7.6,1.6 \mathrm{~Hz}, \mathrm{Ph}-\mathrm{H}), 7.18(1 \mathrm{H}, \mathrm{td}, J=7.5,1.1 \mathrm{~Hz}, \mathrm{Ph}-\mathrm{H}), 7.02(1 \mathrm{H}, \mathrm{d}, J=8.2 \mathrm{~Hz}, \mathrm{Ph}-\mathrm{H}), 3.84$ $\left(3 \mathrm{H}, \mathrm{s}, \mathrm{OCH}_{3}\right), 2.73\left(3 \mathrm{H}, \mathrm{s}, \mathrm{Ph}-\mathrm{CH}_{3}\right) .{ }^{13} \mathrm{C} N M R\left(\mathrm{CD}_{2} \mathrm{Cl}_{2}, 126 \mathrm{MHz}\right) \delta 160.7,159.7,158.3,154.3,148.8,141.7$, 136.6, 134.5, 133.7, 132.8, 131.4, 131.0, 130.6, 129.3, 128.7, 124.7, 124.3, 120.6, 120.4, 110.3, 103.4, 56.2, 23.2. HRMS $\left(\mathrm{ESI}^{+}\right) \mathrm{m} / \mathrm{z}$ calcd for $\mathrm{C}_{24} \mathrm{H}_{20} \mathrm{CIN}_{2} \mathrm{O}_{3} 419.1157$, found $419.1160[\mathrm{M}+\mathrm{H}]^{+}$.

\subsubsection{Synthetic procedure for II-1-II-13}

2-(4-(2-fluorobenzoyl)piperazine-1-carbonyl)-8-methoxy-5-methylquinolin-4(1H)-one (II-1) was synthesized referring to the reported procedures. ${ }^{[19]} \mathrm{A}$ mixture of 2-fluorobenzoic acid $(0.28 \mathrm{~g}, 2.0 \mathrm{mmol}), \mathrm{EDCl}(0.45 \mathrm{~g}$, $2.3 \mathrm{mmol})$ and HOBt $(0.31 \mathrm{~g}, 2.3 \mathrm{mmol})$ in DMF $(10 \mathrm{~mL})$ was stirred for $30 \mathrm{~min}$, followed by the addition of 8-methoxy-5-methyl-2-(piperazine-1-carbonyl)quinolin-4(1H)-one $(0.50 \mathrm{~g}, 1.6 \mathrm{mmol})$. The solution was reacted for $12 \mathrm{~h}$, and then poured into $\mathrm{DCM}(100 \mathrm{~mL})$. The organic phase was washed with water (100 $\mathrm{mL}$ ), and separated by extraction. The resulting organic layer was dried, filtered and concentrated. The obtained residue was purified by chromatograph on silica gel using DCM/methanol $(v / v=100 / 1)$ as eluent to obtain II-1. Compounds II-2 - II-13 were provided in a similar manner.

2-(4-(2-fluorobenzoyl)piperazine-1-carbonyl)-8-methoxy-5-methylquinolin-4(1H)-one (II-1): White solid, yield 55\%, m.p. 208-209 ${ }^{\circ} \mathrm{C} .{ }^{1} \mathrm{H}$ NMR $\left(\mathrm{CDCl}_{3}, 500 \mathrm{MHz}\right) \delta 9.17(1 \mathrm{H}, \mathrm{s}, \mathrm{NH}), 7.48-7.40(2 \mathrm{H}, \mathrm{m}, \mathrm{Ph}-\mathrm{H}), 7.24$ $(1 \mathrm{H}, \mathrm{t}, J=7.5 \mathrm{~Hz}, \mathrm{Ph}-\mathrm{H}), 7.12(1 \mathrm{H}, \mathrm{t}, J=8.9 \mathrm{~Hz}, \mathrm{Ph}-\mathrm{H}), 6.96(1 \mathrm{H}, \mathrm{d}, J=8.2 \mathrm{~Hz}, \mathrm{Ph}-\mathrm{H}), 6.92(1 \mathrm{H}, \mathrm{d}, J=8.1 \mathrm{~Hz}$, Ph-H), $6.25\left(1 \mathrm{H}, \mathrm{s}\right.$, quinolone-H), $3.95\left(3 \mathrm{H}, \mathrm{s}, \mathrm{OCH}_{3}\right), 3.87\left(4 \mathrm{H}, \mathrm{s},\left(\mathrm{CH}_{2}\right)_{2}\right), 3.76\left(2 \mathrm{H}, \mathrm{s}, \mathrm{CH}_{2}\right), 3.44(2 \mathrm{H}, \mathrm{s}$, $\left.\mathrm{CH}_{2}\right), 2.82\left(3 \mathrm{H}, \mathrm{s}, \mathrm{Ph}-\mathrm{CH}_{3}\right) .{ }^{13} \mathrm{C} \mathrm{NMR}\left(\mathrm{CDCl}_{3}, 126 \mathrm{MHz}\right) \delta 180.5,165.4,164.4,159.1,157.1,146.1,138.4$, $132.0(\mathrm{~d}, J=8.2 \mathrm{~Hz}$ ), 131.8 (d, $J=58.3 \mathrm{~Hz}), 129.4$ (d, $J=3.4 \mathrm{~Hz}), 125.4,125.0$ (d, $J=3.4 \mathrm{~Hz}), 124.6,123.2$ (d, $J=17.5 \mathrm{~Hz}), 115.9$ (d, $J=21.4 \mathrm{~Hz}), 111.5,110.6,56.0,46.8,42.0,22.5$. HRMS (ESI $\left.{ }^{+}\right) \mathrm{m} / \mathrm{z}$ calcd for $\mathrm{C}_{23} \mathrm{H}_{23} \mathrm{FN}_{3} \mathrm{O}_{4} 424.1667$, found $424.1671[\mathrm{M}+\mathrm{H}]^{+}$.

8-methoxy-5-methyl-2-(4-(2-(trifluoromethyl)benzoyl)piperazine-1-carbonyl)quinolin-4(1H)-one (II-2): White solid, yield $60 \%$, m.p. $255-257^{\circ} \mathrm{C} .{ }^{1} \mathrm{H}$ NMR $\left(\mathrm{CDCl}_{3}, 500 \mathrm{MHz}\right) \delta 9.28(1 \mathrm{H}, \mathrm{s}, \mathrm{NH}), 7.72(1 \mathrm{H}, \mathrm{d}, J=7.8 \mathrm{~Hz}$, PhH), $7.63(1 \mathrm{H}, \mathrm{t}, J=7.5 \mathrm{~Hz}, \mathrm{Ph}-\mathrm{H}), 7.55(1 \mathrm{H}, \mathrm{t}, J=7.7 \mathrm{~Hz}, \mathrm{Ph}-\mathrm{H}), 7.34(1 \mathrm{H}, \mathrm{d}, J=7.5 \mathrm{~Hz}, \mathrm{Ph}-\mathrm{H}), 6.95(1 \mathrm{H}, \mathrm{d}, J$ $=8.1 \mathrm{~Hz}, \mathrm{Ph}-\mathrm{H}), 6.91(1 \mathrm{H}, \mathrm{d}, J=8.1 \mathrm{~Hz}, \mathrm{Ph}-\mathrm{H}), 6.23(1 \mathrm{H}, \mathrm{s}$, quinolone-H), $4.07-3.97(1 \mathrm{H}, \mathrm{m}$, piperazine-H), $3.96-3.87\left(4 \mathrm{H}, \mathrm{m}, \mathrm{OCH}_{3}\right.$ and piperazine- $\left.\mathrm{H}\right), 3.82-3.67(3 \mathrm{H}, \mathrm{m}$, piperazine- $\mathrm{H}), 3.65-3.57(1 \mathrm{H}, \mathrm{m}$,

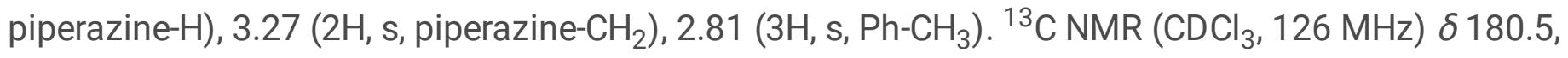
167.6, 164.3, 146.2, 138.6, 134.0, 132.4, 132.1, 131.5, 129.6, 127.2, 126.9 (q, J=32.0 Hz), 126.9 (q, J = 4.8 $\mathrm{Hz}), 125.5,124.5,123.6$ (q, $J=274.2 \mathrm{~Hz}), 111.3,110.6,56.0,46.8,41.6,22.5$. HRMS $\left(\mathrm{ESI}^{+}\right) \mathrm{m} / \mathrm{z}$ calcd for $\mathrm{C}_{24} \mathrm{H}_{23} \mathrm{~F}_{3} \mathrm{~N}_{3} \mathrm{O}_{4} 474.1635$, found $474.1638[\mathrm{M}+\mathrm{H}]^{+}$.

2-(4-(2-chloro-4-(methylsulfonyl)benzoyl)piperazine-1-carbonyl)-8-methoxy-5-methylquinolin-4(1H)-one (II3): White solid, yield 53\%, m.p. $284-286^{\circ} \mathrm{C} .{ }^{1} \mathrm{H}$ NMR (DMSO- $\left.d_{6}, 500 \mathrm{MHz}\right) \delta 11.22$ and $11.18(1 \mathrm{H}, \mathrm{s}, \mathrm{NH})$, 
8.12 and $8.07(1 \mathrm{H}, \mathrm{s}, \mathrm{Ph}-\mathrm{H}), 8.01$ and $7.96(1 \mathrm{H}, \mathrm{d}, J=7.5 \mathrm{~Hz}, \mathrm{Ph}-\mathrm{H}), 7.76$ and $7.72(1 \mathrm{H}, \mathrm{d}, J=7.5 \mathrm{~Hz}, \mathrm{Ph}-\mathrm{H})$, 7.14 and $7.11(1 \mathrm{H}, \mathrm{d}, J=7.5 \mathrm{~Hz}, \mathrm{Ph}-\mathrm{H}), 6.97$ and $6.94(1 \mathrm{H}, \mathrm{d}, J=7.5 \mathrm{~Hz}, \mathrm{Ph}-\mathrm{H}), 5.99$ and $5.95(1 \mathrm{H}, \mathrm{s}$, quinolone-H), 3.97 and $3.94\left(3 \mathrm{H}, \mathrm{s}, \mathrm{OCH}_{3}\right), 3.90-3.42\left(6 \mathrm{H}, \mathrm{m}\right.$, piperazine- $\left.\mathrm{CH}_{2}\right), 3.34-3.15(5 \mathrm{H}, \mathrm{m}$, $\mathrm{SO}_{2} \mathrm{CH}_{3}$ and piperazine- $\mathrm{CH}_{2}$ ), 2.73 and $2.69\left(3 \mathrm{H}, \mathrm{s}, \mathrm{Ph}-\mathrm{CH}_{3}\right) .{ }^{13} \mathrm{C} \mathrm{NMR}\left(\mathrm{DMSO}-d_{6}, 126 \mathrm{MHz}\right) \delta 179.0$, 164.4, 162.7, 146.7, 143.2, 142.6, 140.1, 131.9, 130.1, 129.7, 129.1, 128.0, 126.3, 124.9, 124.0, 111.0, 108.4, 56.0, 46.6, 46.1, 45.9, 45.3, 43.1, 41.4, 41.0, 40.9, 40.4, 22.4. HRMS (ESI $\left.{ }^{+}\right) \mathrm{m} / \mathrm{z}$ calcd for $\mathrm{C}_{24} \mathrm{H}_{25} \mathrm{CIN}_{3} \mathrm{O}_{6} \mathrm{~S} 518.1147$, found $518.1151[\mathrm{M}+\mathrm{H}]^{+}$.

8-methoxy-5-methyl-2-(4-(2-methylbenzoyl)piperazine-1-carbonyl)quinolin-4(7H)-one (II-4): White solid, yield $60 \%$, m.p. $213-214^{\circ} \mathrm{C}$. ${ }^{1} \mathrm{H}$ NMR (DMSO-d $\left.{ }_{6}, 500 \mathrm{MHz}\right) \delta 11.23$ and $11.16(1 \mathrm{H}, \mathrm{s}, \mathrm{NH}), 7.37-7.09(5 \mathrm{H}$, $\mathrm{m}, \mathrm{Ph}-\mathrm{H}), 6.96(1 \mathrm{H}, \mathrm{d}, J=7.9 \mathrm{~Hz}, \mathrm{Ph}-\mathrm{H}), 5.96$ and $5.91(1 \mathrm{H}, \mathrm{s}$, quinolone- $\mathrm{H}), 3.96$ and $3.93\left(3 \mathrm{H}, \mathrm{s}, \mathrm{OCH}_{3}\right)$, $3.78\left(1 \mathrm{H}, \mathrm{s}, \mathrm{CH}_{\mathrm{A}} \mathrm{H}_{\mathrm{B}}\right), 3.69\left(2 \mathrm{H}, \mathrm{s}, \mathrm{CH}_{2}\right), 3.53\left(1 \mathrm{H}, \mathrm{s}, \mathrm{CH}_{\mathrm{A}} \mathrm{H}_{\mathrm{B}}\right), 3.43\left(1 \mathrm{H}, \mathrm{s}, \mathrm{CH}_{\mathrm{A}} \mathrm{H}_{\mathrm{B}}\right), 3.27\left(2 \mathrm{H}, \mathrm{s}, \mathrm{CH}_{2}\right), 3.17(1 \mathrm{H}$, $\left.\mathrm{s}, \mathrm{CH}_{\mathrm{A}} \mathrm{H}_{\mathrm{B}}\right), 2.71$ and $2.67\left(3 \mathrm{H}, \mathrm{s}, \mathrm{Ph}-\mathrm{CH}_{3}\right), 2.24$ and $2.22\left(3 \mathrm{H}, \mathrm{s}, \mathrm{Ph}_{-} \mathrm{CH}_{3}\right) \cdot{ }^{13} \mathrm{C}$ NMR (DMSO- $\left.d_{6}, 126 \mathrm{MHz}\right) \delta$ 179.6, 169.3, 163.3, 147.3, 143.9, 139.7, 136.5, 134.24, 132.4, 130.7, 130.1, 129.3, 126.2, 125.4, 124.6, 111.6, 108.9, 56.6, 47.3, 46.9, 46.5, 45.9, 42.1, 41.6, 41.2, 23.0, 19.1. HRMS $\left(\mathrm{ESI}^{+}\right) \mathrm{m} / \mathrm{z}$ calcd for $\mathrm{C}_{24} \mathrm{H}_{26} \mathrm{~N}_{3} \mathrm{O}_{4} 420.1918$, found $420.1917[\mathrm{M}+\mathrm{H}]^{+}$.

8-methoxy-2-(4-(2-methoxybenzoyl)piperazine-1-carbonyl)-5-methylquinolin-4(1H)-one (II-5): White solid, yield $62 \%$, m.p. $241-242^{\circ} \mathrm{C} .{ }^{1} \mathrm{H}$ NMR (DMSO-d $\left.6,500 \mathrm{MHz}\right) \delta 11.24$ and $11.19(1 \mathrm{H}, \mathrm{s}, \mathrm{NH}), 7.43$ and 7.37 $(1 \mathrm{H}, \mathrm{t}, J=7.5 \mathrm{~Hz}, \mathrm{Ph}-\mathrm{H}), 7.23$ and $7.20(1 \mathrm{H}, \mathrm{d}, J=7.0 \mathrm{~Hz}, \mathrm{Ph}-\mathrm{H}), 7.16-6.92(4 \mathrm{H}, \mathrm{m}, \mathrm{Ph}-\mathrm{H}), 5.97$ and 5.93 $(1 \mathrm{H}, \mathrm{s}$, quinolone- $\mathrm{H}), 3.96$ and $3.93\left(3 \mathrm{H}, \mathrm{s}, \mathrm{OCH}_{3}\right), 3.83$ and $3.78\left(3 \mathrm{H}, \mathrm{s}, \mathrm{OCH}_{3}\right), 3.73-3.51(4 \mathrm{H}, \mathrm{m}$, piperazine- $\left.\mathrm{CH}_{2}\right), 3.41\left(1 \mathrm{H}, \mathrm{s}\right.$, piperazine- $\left.\mathrm{CH}_{2}\right), 3.33-3.08\left(3 \mathrm{H}, \mathrm{m}\right.$, piperazine- $\left.\mathrm{CH}_{2}\right), 2.72$ and $2.68(3 \mathrm{H}, \mathrm{s}$, Ph- $\mathrm{CH}_{3}$ ). ${ }^{13} \mathrm{C}$ NMR (DMSO- $\left.d_{6}, 126 \mathrm{MHz}\right) \delta 179.6,167.1,163.2,147.3,143.9,140.1,135.1,132.4,131.0$, 130.2, 129.3, 128.3, 125.7, 124.6, 121.2, 111.9, 108.9, 56.6, 56.0, 47.3, 46.9, 46.6, 45.9, 42.1, 41.7, 41.4, 40.8, 23.0. HRMS $\left(\mathrm{ESI}^{+}\right) \mathrm{m} / \mathrm{z}$ calcd for $\mathrm{C}_{24} \mathrm{H}_{26} \mathrm{~N}_{3} \mathrm{O}_{5} 436.1867$, found $436.1866[\mathrm{M}+\mathrm{H}]^{+}$.

8-methoxy-5-methyl-2-(4-(2-methyl-4-(trifluoromethyl)thiazole-5-carbonyl)piperazine-1-carbonyl)quinolin4(1H)-one (II-6): White solid, yield 66\%, m.p. $225-257^{\circ} \mathrm{C} .{ }^{1} \mathrm{H} \mathrm{NMR}\left(\mathrm{CDCl}_{3}, 500 \mathrm{MHz}\right) \delta 9.34(1 \mathrm{H}, \mathrm{s}, \mathrm{NH})$, $6.96(1 \mathrm{H}, \mathrm{d}, J=8.2 \mathrm{~Hz}, \mathrm{Ph}-\mathrm{H}), 6.92(1 \mathrm{H}, \mathrm{d}, J=8.1 \mathrm{~Hz}, \mathrm{Ph}-\mathrm{H}), 6.24\left(1 \mathrm{H}, \mathrm{s}\right.$, quinolone-H), $3.93\left(3 \mathrm{H}, \mathrm{s}, \mathrm{OCH}_{3}\right)$, $3.90-3.69\left(6 \mathrm{H}, \mathrm{m}\right.$, piperazine- $\left.\mathrm{CH}_{2}\right), 3.43\left(2 \mathrm{H}\right.$, s, piperazine- $\left.\mathrm{CH}_{2}\right), 2.81\left(3 \mathrm{H}, \mathrm{s}\right.$, thiazole- $\left.\mathrm{CH}_{3}\right), 2.77(3 \mathrm{H}, \mathrm{s}$, $\left.\mathrm{Ph}-\mathrm{CH}_{3}\right) .{ }^{13} \mathrm{C} \mathrm{NMR}\left(\mathrm{CDCl}_{3}, 126 \mathrm{MHz}\right) \delta 180.49,168.39,164.38,159.51,146.18,139.90$ (q, $\left.J=36.7 \mathrm{~Hz}\right)$, 138.5, 132.1, 131.5, 131.0, 125.5, 124.6, 120.1 (q, $J=272.4 \mathrm{~Hz}$ ), 111.3, 110.7, 56.0, 47.1, 42.2, 22.5, 19.1 . HRMS (ESI $\left.{ }^{+}\right) \mathrm{m} / \mathrm{z}$ calcd for $\mathrm{C}_{22} \mathrm{H}_{22} \mathrm{~F}_{3} \mathrm{~N}_{4} \mathrm{O}_{4} \mathrm{~S} 495.1308$, found $495.1311[\mathrm{M}+\mathrm{H}]^{+}$.

8-methoxy-5-methyl-2-(4-(4-methyl-1,2,3-thiadiazole-5-carbonyl)piperazine-1-carbonyl)quinolin-4(1H)-one (II-7): White solid, yield 64\%, m.p. $211-213^{\circ} \mathrm{C} .{ }^{1} \mathrm{H} \mathrm{NMR}\left(\mathrm{CDCl}_{3}, 500 \mathrm{MHz}\right) \delta 9.29(1 \mathrm{H}, \mathrm{s}, \mathrm{NH}), 6.97(1 \mathrm{H}, \mathrm{d}, J$ $=8.2 \mathrm{~Hz}, \mathrm{Ph}-\mathrm{H}), 6.93(1 \mathrm{H}, \mathrm{d}, J=8.1 \mathrm{~Hz}, \mathrm{Ph}-\mathrm{H}), 6.24(1 \mathrm{H}, \mathrm{s}$, quinolone $-\mathrm{H}), 3.94\left(3 \mathrm{H}, \mathrm{s}, \mathrm{OCH}_{3}\right), 3.90-3.15$

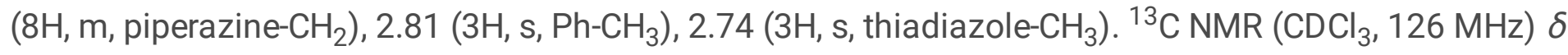


$180.5,164.4,160.5,157.5,146.2,141.7,138.3,132.1,131.5,125.6,124.6,111.4,110.7,56.0,46.8,42.5$, 22.5, 13.0. HRMS $\left(\mathrm{ESI}^{+}\right) \mathrm{m} / \mathrm{z}$ calcd for $\mathrm{C}_{20} \mathrm{H}_{22} \mathrm{~N}_{5} \mathrm{O}_{4} \mathrm{~S} 428.1387$, found $428.1389[\mathrm{M}+\mathrm{H}]^{+}$.

2-(4-(3,4-dichloroisothiazole-5-carbonyl)piperazine-1-carbonyl)-8-methoxy-5-methylquinolin-4(1H)-one (II8): White solid, yield 58\%, m.p. $185-186^{\circ} \mathrm{C} .{ }^{1} \mathrm{H}$ NMR $\left(\mathrm{CDCl}_{3}, 500 \mathrm{MHz}\right) \delta 9.35(1 \mathrm{H}, \mathrm{s}, \mathrm{NH}), 6.97(1 \mathrm{H}, \mathrm{d}, J=$ $8.2 \mathrm{~Hz}, \mathrm{Ph}-\mathrm{H}), 6.93(1 \mathrm{H}, \mathrm{d}, J=8.1 \mathrm{~Hz}, \mathrm{Ph}-\mathrm{H}), 6.25\left(1 \mathrm{H}, \mathrm{s}\right.$, quinolone-H), $3.93\left(3 \mathrm{H}, \mathrm{s}, \mathrm{OCH}_{3}\right), 3.90-3.35(8 \mathrm{H}$, $\left.\mathrm{m},\left(\mathrm{CH}_{2}\right)_{4}\right), 2.81\left(3 \mathrm{H}, \mathrm{s}, \mathrm{Ph}-\mathrm{CH}_{3}\right) .{ }^{13} \mathrm{C} \mathrm{NMR}\left(\mathrm{CDCl}_{3}, 126 \mathrm{MHz}\right) \delta 180.5,164.4,158.7,154.5,148.5,146.2$, 138.5, 132.1, 131.5, 125.6, 124.6, 120.5, 111.3, 110.7, 56.0, 46.9, 42.5, 22.5. HRMS (ESI ${ }^{+}$) m/z calcd for $\mathrm{C}_{20} \mathrm{H}_{19} \mathrm{Cl}_{2} \mathrm{~N}_{4} \mathrm{O}_{4} \mathrm{~S} 481.0499$, found $481.0501[\mathrm{M}+\mathrm{H}]^{+}$.

2-(4-(4-chloro-3-ethyl-1-methyl-1H-pyrazole-5-carbonyl)piperazine-1-carbonyl)-8-methoxy-5-methylquinolin4(1H)-one (II-9): White solid, yield $57 \%$, m.p. $186-187^{\circ} \mathrm{C} .{ }^{1} \mathrm{H}$ NMR (DMSO- $\left.d_{6}, 500 \mathrm{MHz}\right) \delta 11.30-11.15$ $(1 \mathrm{H}, \mathrm{s}, \mathrm{NH}), 7.13(1 \mathrm{H}, \mathrm{s}, \mathrm{Ph}-\mathrm{H}), 6.96(1 \mathrm{H}, \mathrm{s}, \mathrm{Ph}-\mathrm{H}), 5.98(1 \mathrm{H}, \mathrm{s}$, quinolone- $\mathrm{H}), 3.96\left(3 \mathrm{H}, \mathrm{s}, \mathrm{OCH}_{3}\right), 3.85-3.65$ $\left(7 \mathrm{H}, \mathrm{m}\right.$, pyrazole- $\mathrm{CH}_{3}$ and $\left.\left(\mathrm{CH}_{2}\right)_{2}\right), 3.58-3.39\left(4 \mathrm{H}, \mathrm{m},\left(\mathrm{CH}_{2}\right)_{2}\right), 2.72\left(3 \mathrm{H}, \mathrm{s}, \mathrm{Ph}_{-} \mathrm{CH}_{3}\right), 2.65-2.52(2 \mathrm{H}, \mathrm{m}$, $\left.\mathrm{CH}_{2} \mathrm{CH}_{3}\right), 1.25-1.13\left(3 \mathrm{H}, \mathrm{m}, \mathrm{CH}_{2} \mathrm{CH}_{3}\right) .{ }^{13} \mathrm{C}$ NMR (DMSO-d $\left.d_{6}, 126 \mathrm{MHz}\right) \delta 179.6,163.3,159.1,148.8,147.3$, 143.7, 133.9, 132.4, 130.2, 125.4, 124.6, 111.6, 109.0, 105.8, 56.6, 47.6, 46.7, 46.1, 42.4, 42.0, 41.5, 38.5, $22.9,19.0,13.1$. HRMS $\left(E S I^{+}\right) \mathrm{m} / \mathrm{z}$ calcd for $\mathrm{C}_{23} \mathrm{H}_{27} \mathrm{ClN}_{5} \mathrm{O}_{4} 472.1746$, found $472.1746[\mathrm{M}+\mathrm{H}]^{+}$.

8-methoxy-5-methyl-2-(4-(1-methyl-3-(trifluoromethyl)-1H-pyrazole-4-carbonyl)piperazine-1carbonyl)quinolin-4(1H)-one (II-10): White solid, yield 60\%, m.p. $221-222^{\circ} \mathrm{C} .{ }^{1} \mathrm{H}$ NMR (DMSO- $d_{6}, 500$ $\mathrm{MHz}) \delta 11.20(1 \mathrm{H}, \mathrm{s}, \mathrm{NH}), 8.16(1 \mathrm{H}, \mathrm{s}$, pyrazole-H), $7.13(1 \mathrm{H}, \mathrm{d}, J=7.9 \mathrm{~Hz}, \mathrm{Ph}-\mathrm{H}), 6.96(1 \mathrm{H}, \mathrm{d}, J=7.8 \mathrm{~Hz}$, Ph-H), $5.96\left(1 \mathrm{H}, \mathrm{s}\right.$, quinolone-H), $3.95\left(6 \mathrm{H}, \mathrm{s}, \mathrm{OCH}_{3}\right.$ and pyrazole- $\left.\mathrm{CH}_{3}\right), 3.59\left(6 \mathrm{H}, \mathrm{s},\left(\mathrm{CH}_{2}\right)_{3}\right), 3.34(2 \mathrm{H}, \mathrm{s}$, $\mathrm{CH}_{2}$ ), $2.70\left(3 \mathrm{H}, \mathrm{s}, \mathrm{Ph}-\mathrm{CH}_{3}\right) .{ }^{13} \mathrm{C}$ NMR (DMSO-d $\left.{ }_{6}, 126 \mathrm{MHz}\right) \delta 179.6,163.3,161.6,147.3,143.8,138.2(\mathrm{q}, \mathrm{J}=$ $36.9 \mathrm{~Hz}), 133.0,132.4,130.2,125.4,124.6,121.5$ (q, $J=269.39 \mathrm{~Hz}), 115.2,111.6,109.0,56.6,47.0,41.8$, 22.9. HRMS (ESI ${ }^{+}$) $\mathrm{m} / \mathrm{z}$ calcd for $\mathrm{C}_{22} \mathrm{H}_{23} \mathrm{~F}_{3} \mathrm{~N}_{5} \mathrm{O}_{4} 478.1697$, found $478.1694[\mathrm{M}+\mathrm{H}]^{+}$.

2-(4-(3-(difluoromethyl)-1-methyl-1H-pyrazole-4-carbonyl)piperazine-1-carbonyl)-8-methoxy-5methylquinolin-4(1H)-one (II-11): White solid, yield 62\%, m.p. 201-203 ${ }^{\circ} \mathrm{C} .{ }^{1} \mathrm{H}$ NMR (DMSO- $\left.d_{6}, 500 \mathrm{MHz}\right) \delta$ $11.24(1 \mathrm{H}, \mathrm{s}, \mathrm{NH}), 8.13\left(1 \mathrm{H}, \mathrm{s}\right.$, pyrazole-H), $7.13(1 \mathrm{H}, \mathrm{d}, J=8.1 \mathrm{~Hz}, \mathrm{Ph}-\mathrm{H}), 7.07\left(1 \mathrm{H}, \mathrm{t}, J=54.5 \mathrm{~Hz}, \mathrm{CHF}_{2}\right)$, $6.97(1 \mathrm{H}, \mathrm{d}, J=7.7 \mathrm{~Hz}, \mathrm{Ph}-\mathrm{H}), 5.97\left(1 \mathrm{H}, \mathrm{s}\right.$, quinolone-H), $3.95\left(3 \mathrm{H}, \mathrm{s}, \mathrm{OCH}_{3}\right), 3.90\left(3 \mathrm{H}, \mathrm{s}\right.$, pyrazole $\left.-\mathrm{CH}_{3}\right), 3.67$ - $3.59\left(6 \mathrm{H}, \mathrm{m},\left(\mathrm{CH}_{2}\right)_{3}\right), 3.38\left(2 \mathrm{H}, \mathrm{s}, \mathrm{CH}_{2}\right), 2.71\left(3 \mathrm{H}, \mathrm{s}, \mathrm{Ph}-\mathrm{CH}_{3}\right) .{ }^{13} \mathrm{C}$ NMR (DMSO-d $\left.d_{6}, 126 \mathrm{MHz}\right) \delta 179.6$, 163.3, 162.4, 147.3, $144.3(\mathrm{t}, J=25.3 \mathrm{~Hz}), 143.9,132.7,132.4,130.2,125.4,124.6,114.7,111.5,110.9(\mathrm{t}, J$ $=234.4 \mathrm{~Hz}), 109.0,56.6,47.0,41.8,39.5,22.9$. HRMS $\left(\mathrm{ESI}^{+}\right) \mathrm{m} / \mathrm{z}$ calcd for $\mathrm{C}_{22} \mathrm{H}_{24} \mathrm{~F}_{2} \mathrm{~N}_{5} \mathrm{O}_{4} 460.1791$, found $460.1789[\mathrm{M}+\mathrm{H}]^{+}$.

(E)-2,6-dimethoxy-4-(3-(4-(8-methoxy-5-methyl-4-oxo-1,4-dihydroquinoline-2-carbonyl)piperazin-1-yl)-3oxoprop-1-en-1-yl)phenyl acetate (II-12): White solid, yield 55\%, m.p. $173-174^{\circ} \mathrm{C} .{ }^{1} \mathrm{H} \mathrm{NMR}\left(\mathrm{CDCl}_{3}, 500\right.$ $\mathrm{MHz}) \delta 9.30(1 \mathrm{H}, \mathrm{s}, \mathrm{NH}), 7.62(1 \mathrm{H}, \mathrm{d}, J=15.3 \mathrm{~Hz}, \mathrm{CH}), 6.96(1 \mathrm{H}, \mathrm{d}, J=8.1 \mathrm{~Hz}, \mathrm{Ph}-\mathrm{H}), 6.92(1 \mathrm{H}, \mathrm{d}, J=8.1 \mathrm{~Hz}$, 
Ph-H), $6.79(1 \mathrm{H}, \mathrm{d}, J=15.3 \mathrm{~Hz}, \mathrm{CH}), 6.76(2 \mathrm{H}, \mathrm{s}, \mathrm{Ph}-\mathrm{H}), 6.27\left(1 \mathrm{H}, \mathrm{s}\right.$, quinolone-H), $3.94\left(3 \mathrm{H}, \mathrm{s}, \mathrm{OCH}_{3}\right), 3.84$ $\left(6 \mathrm{H}, \mathrm{s}, \mathrm{OCH}_{3}\right), 3.80\left(8 \mathrm{H}, \mathrm{s}\right.$, piperazine- $\left.\mathrm{CH}_{2}\right), 2.82\left(3 \mathrm{H}, \mathrm{s}, \mathrm{COCH}_{3}\right), 2.33\left(3 \mathrm{H}, \mathrm{s}, \mathrm{Ph}_{-} \mathrm{CH}_{3}\right) \cdot{ }^{13} \mathrm{C} \mathrm{NMR}\left(\mathrm{CDCl}_{3}, 126\right.$ $\mathrm{MHz}) \delta 180.6,168.6,165.5,164.2,152.4,146.2,143.7,138.7,133.2,132.1,131.5,130.2,125.5,124.5$, $116.5,111.4,110.7,104.6,56.3,56.0,45.4,42.0,22.5,20.4$. HRMS $\left(E S I^{+}\right) \mathrm{m} / \mathrm{z}$ calcd for $\mathrm{C}_{29} \mathrm{H}_{32} \mathrm{~N}_{3} \mathrm{O}_{8}$ 550.2184 , found $550.2188[\mathrm{M}+\mathrm{H}]^{+}$.

(E)-6-methoxy-5-(6-(4-(8-methoxy-5-methyl-4-oxo-1,4-dihydroquinoline-2-carbonyl)piperazin-1-yl)-3-methyl6-oxohex-2-en-1-yl)-7-methyl-3-oxo-1,3-dihydroisobenzofuran-4-yl acetate (II-13): White solid, yield 47\%, m.p. $153-154^{\circ} \mathrm{C} .{ }^{1} \mathrm{H} \mathrm{NMR}\left(\mathrm{CDCl}_{3}, 500 \mathrm{MHz}\right) \delta 9.16(1 \mathrm{H}, \mathrm{s}, \mathrm{NH}), 6.97(1 \mathrm{H}, \mathrm{d}, J=8.1 \mathrm{~Hz}, \mathrm{Ph}-\mathrm{H}), 6.93(1 \mathrm{H}, \mathrm{d}$, $J=8.1 \mathrm{~Hz}, \mathrm{Ph}-\mathrm{H}), 6.25(1 \mathrm{H}, \mathrm{s}$, quinolone-H), $5.16(2 \mathrm{H}, \mathrm{s}$, isobenzofuran-1(3H)-one-CH$), 5.07(1 \mathrm{H}, \mathrm{t}, J=6.4$ $\mathrm{Hz}, \mathrm{CH}), 3.96\left(3 \mathrm{H}, \mathrm{s}, \mathrm{OCH}_{3}\right), 3.80\left(3 \mathrm{H}, \mathrm{s}, \mathrm{OCH}_{3}\right), 3.75-3.63\left(6 \mathrm{H}, \mathrm{m}\right.$, piperazine- $\left.\mathrm{CH}_{2}\right), 3.52(2 \mathrm{H}, \mathrm{s}$, piperazine- $\left.\mathrm{CH}_{2}\right), 3.37\left(2 \mathrm{H}, \mathrm{d}, J=6.6 \mathrm{~Hz}, \mathrm{Ph}-\mathrm{CH}_{2}\right), 2.82\left(3 \mathrm{H}, \mathrm{s}, \mathrm{Ph}-\mathrm{CH}_{3}\right), 2.43-2.37\left(5 \mathrm{H}, \mathrm{m}, \mathrm{COCH}_{3}\right.$ and $\left.\mathrm{CH}_{2}\right), 2.34-2.29\left(2 \mathrm{H}, \mathrm{m}, \mathrm{CH}_{2}\right), 2.23\left(3 \mathrm{H}, \mathrm{s}, \mathrm{Ph}-\mathrm{CH}_{3}\right), 1.81\left(3 \mathrm{H}, \mathrm{s}, \mathrm{CH}_{3}\right) .{ }^{13} \mathrm{C} \mathrm{NMR}\left(\mathrm{CDCl}_{3}, 126 \mathrm{MHz}\right) \delta 180.6$, 171.2, 169.0, 168.3, 164.3, 162.6, 146.3, 146.2, 145.9, 138.5, 134.9, 132.1, 131.6, 129.2, 125.5, 124.6, 123.0, 122.0, 113.5, 111.5, 110.7, 68.4, 61.3, 56.0, 45.4, 41.4, 34.4, 31.6, 23.5, 22.5, 20.6, 16.6, 11.8. HRMS $\left(\mathrm{ESI}^{+}\right) \mathrm{m} / \mathrm{z}$ calcd for $\mathrm{C}_{35} \mathrm{H}_{40} \mathrm{~N}_{3} \mathrm{O}_{9} 646.2759$, found $646.2762[\mathrm{M}+\mathrm{H}]^{+}$.

\subsection{Fungicidal activity measurement}

With fluopyram and carbendazim as positive controls, the mycelial growth inhibition method was used to determine the in vitro inhibitory activities of the target molecules against common agricultural pathogens according to the previously reported procedures. ${ }^{22}$ The tested pathogens include Rhizoctonia solani (RS), Gibberella zeae (GZ), Botrytis cinerea (BC), Physalospora piricola (PP), Cercospora circumscissa Sacc. (CS), Colletotrichum capsici (CC), Alternaria kikuchiana Tanaka (AK), and Alternaria sp. (AS).

\subsection{In vitro Cellular Cytotoxicity Assays}

The in vitro cell viability of hepatocellular carcinoma cell lines (HepG-2) was assessed by MTT colorimetric assay according to the reported methods. ${ }^{23,24}$ Firstly, cells were seeded in 96-well plates, incubated in a $\mathrm{CO}_{2}$ incubator at $37^{\circ} \mathrm{C}$ for $24 \mathrm{~h}$, and then treated with freshly prepared culture mediums containing the tested compounds $(100 \mu \mathrm{M})$ for $24 \mathrm{~h}$. Secondly, a fresh solution of MTT $(5 \mathrm{mg} / \mathrm{ml})$ was added to each single well of the 96-well plate, which was further incubated in a $\mathrm{CO}_{2}$ incubator for another $4 \mathrm{~h}$. After removal of the medium, the cells were dissolved with $100 \mu \mathrm{L}$ of DMSO and analyzed in a multiwall-plate reader (Bio-Rad iMark) at $490 \mathrm{~nm}$.

\subsection{Fluorescence characteristics determination \\ 4.5.1 Fluorescence characteristics measurement}

The UV-Vis absorption spectra was firstly measured from $200 \mathrm{~nm}$ to $800 \mathrm{~nm}$ at $50 \mu \mathrm{M}$ to determine the appropriate excitation wavelength. The fluorescence emission spectra were provided with EM slit of $5 \mathrm{~nm}$, PMT voltage of $480 \mathrm{~V}$ at the same concentration $(50 \mu \mathrm{M})$. Subsequently, the absolute fluorescence 
quantum yields (QY) were recorded on a FLS1000 spectrometer with the parameters referred to the obtained fluorescence emission and excitation spectra.

\subsubsection{TD-DFT calculation}

The singlet ground-states geometrical optimizations were performed, and the calculations were carried out by using spin-restricted DFT method with B3LYP, ${ }^{25-28}$ in conjunction with $6-31+G(d, p)$ basis set. Based on the optimized geometries of the molecules, the molecular orbitals (MOs) were calculated at the same level. The HOMO energy ( $\left.\mathrm{E}_{\mathrm{HOMO}}\right)$ of each compounds are taken from the eigenvalues of the KohnSham calculated from the DFT. TD-DFT calculation of the single excitation energies were performed at the ground states and the calculations using B3LYP, and the basis set is $6-31+G(d, p)$. Then the energy gaps $\left(E_{g}\right)$ were estimated based on the single-singlet electronic transition energies. The LUMO energy level $\left(E_{\text {LUMO }}\right)$ can be got according the equation of $E_{\text {LUMO }}=E_{\text {HOMO }}(D F T)+E_{g}(T D D F T)$. The $E_{L U M O}$ got from this way are excellent agreement with the experiments for the compounds. ${ }^{29}$ All the calculations of both ground and excited states were performed within the Gaussian 09 quantum chemical package. ${ }^{[30]}$

\section{Declarations}

\section{Acknowledgments}

This research was supported by the National Natural Science Foundation of China (No. 32001929), the National Innovation and Entrepreneurship Training Program for College Students (No. 202110447013, 202110447032), and the Innovation and Entrepreneurship Training Program for College Students of Liaocheng University (No. CXCY2020Y116).

\section{Conflict of interest}

There are no conflicts of interest to declare.

\section{References}

1. Kabbage M, Piotrowski JS, Thill E, Westrick NM, Ralph J, Hockemeyer K, Koch PL (2020) Plant Pathol 69:112

2. Zhu JK, Gao JM, Yang CJ, Shang XF, Zhao ZM, Lawoe RK, Zhou R, Sun Y, Yin XD, Liu YQ (2020) J Agric Food Chem 68:2306

3. Zheng JG, Liu TT, Guo ZX, Zhang L, Mao LG, Zhang YN, Jiang HY (2019) Sci Rep-UK 9:1

4. Song PP, Zhao J, Liu ZL, Duan YB, Hou YP, Zhao CQ, Wu M, Wei M, Wang NH, Lv Y, Han ZJ (2017) Pest Manag Sci 73:94

5. Zhang ZL, Xie YJ, Hu X, Shi HA, Wei M, Lin ZF (2018) Nat Prod Commun 13:1721

6. Hasheminejad N, Khodaiyan F, Safari M (2019) Food Chem 275:113 
7. Yang GZ, Zhu JK, Yin XD, Yan YF, Wang YL, Shang XF, Liu YQ, Zhao ZM, Peng JW, Liu H (2019) J Agric Food Chem 67:11340

8. Li JW, Vederas JC (2009) Science 325:161

9. Rodrigues T, Reker D, Petra Schneider P, Schneider G (2016) Nat Chem 8:531

10. Newman DJ, Cragg GM (2020) J Nat Prod 83:770

11. Liu J, Lu SC, Feng JY, Li CK, Wang WL, Pei YM, Ding SL, Zhang M, Li HL, Na RS, Li QX (2020) J Agric Food Chem 68:2116

12. Jadulco RC, Pond CD, Van Wagoner RM, Koch M, Gideon OG, Matainaho TK, Piskaut P, Barrows LR (2014) J Nat Prod 77:183

13. Cretton S, Dorsaz S, Azzollini A, Favre-Godal Q, Marcourt L, Ebrahimi SN, Voinesco F, Michellod E, Sanglard D, Gindro K, Wolfender JL, Cuendet M, Christen P (2016) J Nat Prod 79:300

14. Dorsaz S, Snäkä T, Favre-Godal Q, Maudens P, Boulens N, Furrer P, Ebrahimi SN, Hamburger M, Allémann E, Gindro K, Queiroz EF, Riezman H, Wolfender JL, Sanglard D (2017) Antimicrob. Agents Ch. 61, e00829-17/1

15. Hua XW, Liu WR, Chen Y, Ru J, Guo SJ, Yu XB, Cui YH, Liu XH, Gu YC, Xue CM, Liu Y, Sui JK, Wang GQ (2021) J Agric Food Chem 69:11470

16. Hua X, Liu N, Zhou S, Zhang L, Yin H, Wang G, Fan Z, Ma Y (2020) Engineering 6:553

17. Hua X, Liu N, Fan Z, Zong G, Ma Y, Lei K, Yin H, Wang G (2019) Chin J Org Chem 39:2581

18. Hua X, Liu W, Su Y, Liu X, Liu J, Liu N, Wang G, Jiao X, Fan X, Xue C, Liu Y, Liu M (2020) Pest Manag Sci 76:2368

19. Liu WR, Hua XW, Zhou S, Yuan FY, Wang GQ, Liu Y, Xing XR (2021) Chinese J Struct Chem 40:666

20. Wang BL, Li ZM, Zhang Y, Zhang LY, Zhang X, Li YH (2017) CN 105541748,

21. Bink A, Govaert G, François I, Pellens K, Meerpoel L, Borgers M, Minnebruggen GV, Vroome V, Cammue B, Thevissen K (2010) FEMS Yeast Res 10:812

22. Fan ZJ, Yang ZK, Zhang HK, Mi N, Wang H, Cai F, Zuo X, Zheng QX, Song HB (2010) J Agric Food Chem 58:2630

23. An BH, Zhang RF, Li QL, Du XM, Ru J, Zhang SL, Ma CL (2019) J Organomet Chem 881:51

24. Li LM, Chen Y, Wang QP, Li ZJ, Liu ZF, Hua XW, Han J, Chang CX, Wang ZP, Li DC (2021) Int J Nanomed 16:5513

25. Beck AD (1993) J Chem Phys $98: 1372$

26. Beck AD (1993) J Chem Phys 98:5648

27. Yanai T, Tew DP, Handy NC (2004) Chem Phys Lett 393:51

28. Hertwing RH, Koch W (1997) Chem Phys Lett 268:345

29. Ku J, Lansac Y, Jang YH (2011) J Phys Chem C 115:21508

30. Frisch MJ, Trucks GW, Schlegel HB, Scuseria GE, Robb MA et al (2009)Gaussian, Inc., Wallingford CT, 


\section{Schemes 1}

Schemes 1 is available in the Supplemental Files section

\section{Figures}

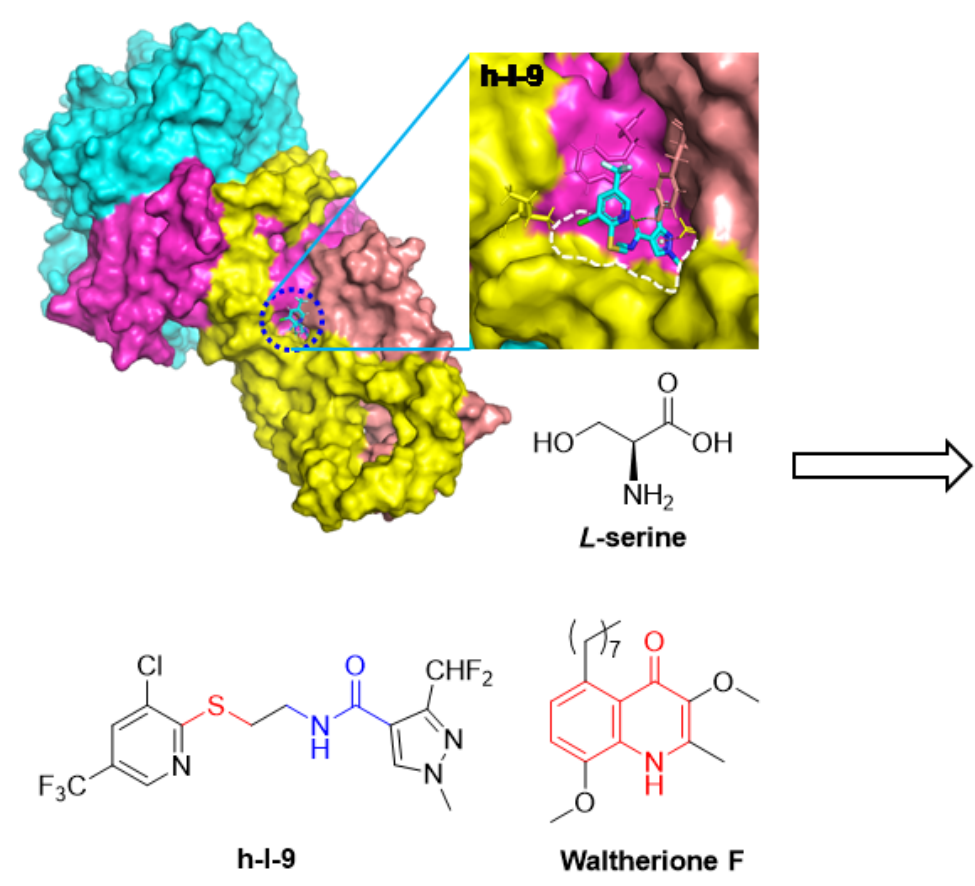

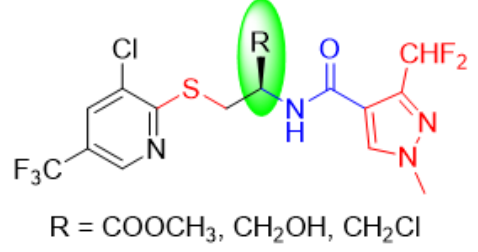

X-I<smiles>[R]c1[nH]c2c(OC)ccc(C)c2c(=O)c1[R]</smiles>

QD<smiles>[R]c1nc2c(OC)ccc(C)c2c(OC(=O)c2cn(C)nc2C(F)F)c1[R]</smiles>

X-II
In vilto inhbition activity against Aftemaria sp. SDH

Compounds $\operatorname{lc}_{50}\left(\operatorname{gg} L^{-1}\right)$

\begin{tabular}{|c|c|}
\hline Fluopyram & 13.36 \\
\hline$X+3$ & 18.32 \\
\hline$X+4$ & 6.81 \\
\hline
\end{tabular}

Molecular docking with SDH (PDB code:4YTP)

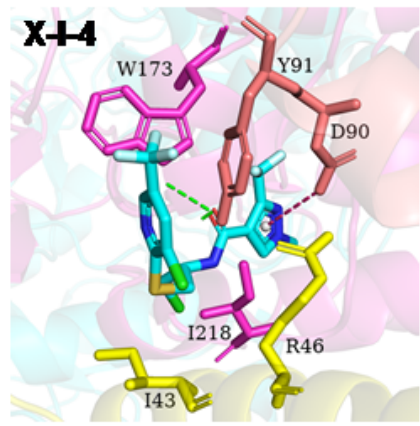

\section{Figure 1}

The molecules obtained in the previous work<smiles>Cn1cc(C(=O)N[C@@H](CCl)CSc2ncc(C(F)(F)F)cc2Cl)c(C(F)F)n1</smiles>

$\mathrm{X}-\mathrm{I}-4$<smiles>COC(=O)c1cc(=O)c2c(C)ccc(OC)c2[nH]1</smiles>

QD-1<smiles></smiles>

$X-11-5$<smiles>COc1ccc(C)c2c(=O)cc(C(=O)NC3CCCCC3)[nH]c12</smiles>

Target compound I<smiles>[R]c1nn(CN2CCN([R])CC2)c(=S)o1</smiles>

P-1<smiles>COc1nc2ccc(CN3CCN(C(=N)N)CC3)cc2cc1Cc1ccc(Cl)cc1</smiles>

P-2<smiles>COc1ccc(C)c2c(=O)cc(C(=O)N3CCN(C(=O)[Al])CC3)[nH]c12</smiles>

Target compound II

Figure 2

Molecular design strategy in this project 
A

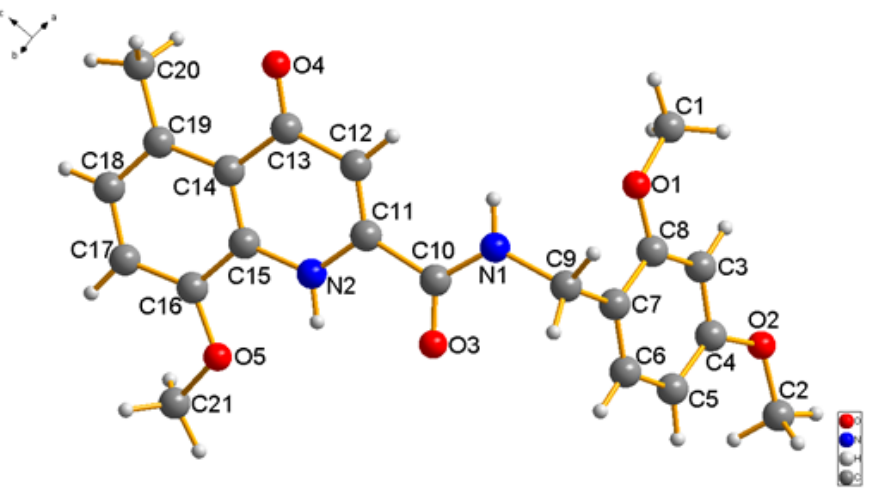

B!

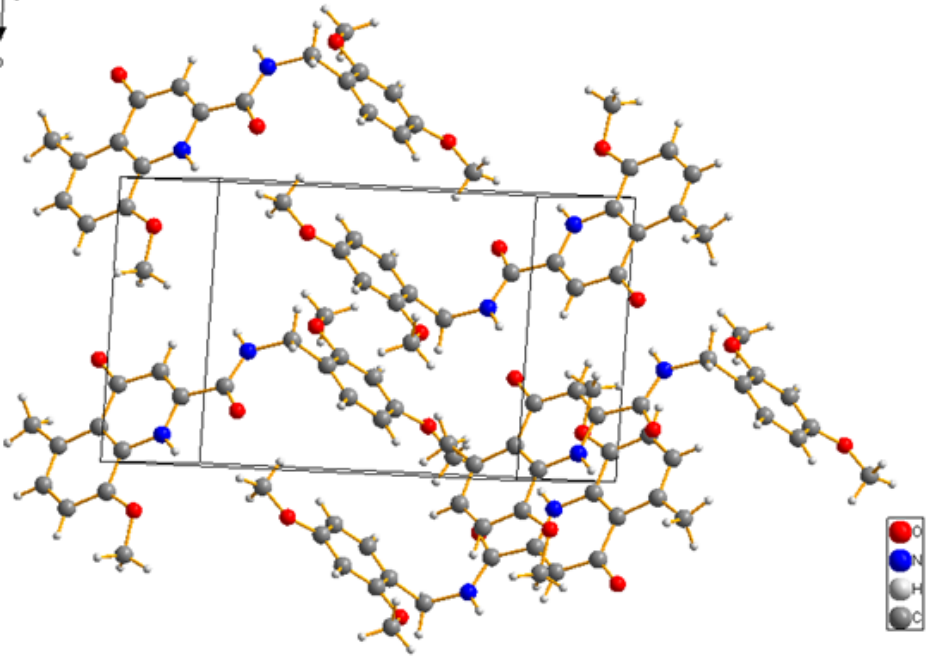

Figure 3

The crystal structure $(A)$ and packing $(B)$ of the target compound I-6 


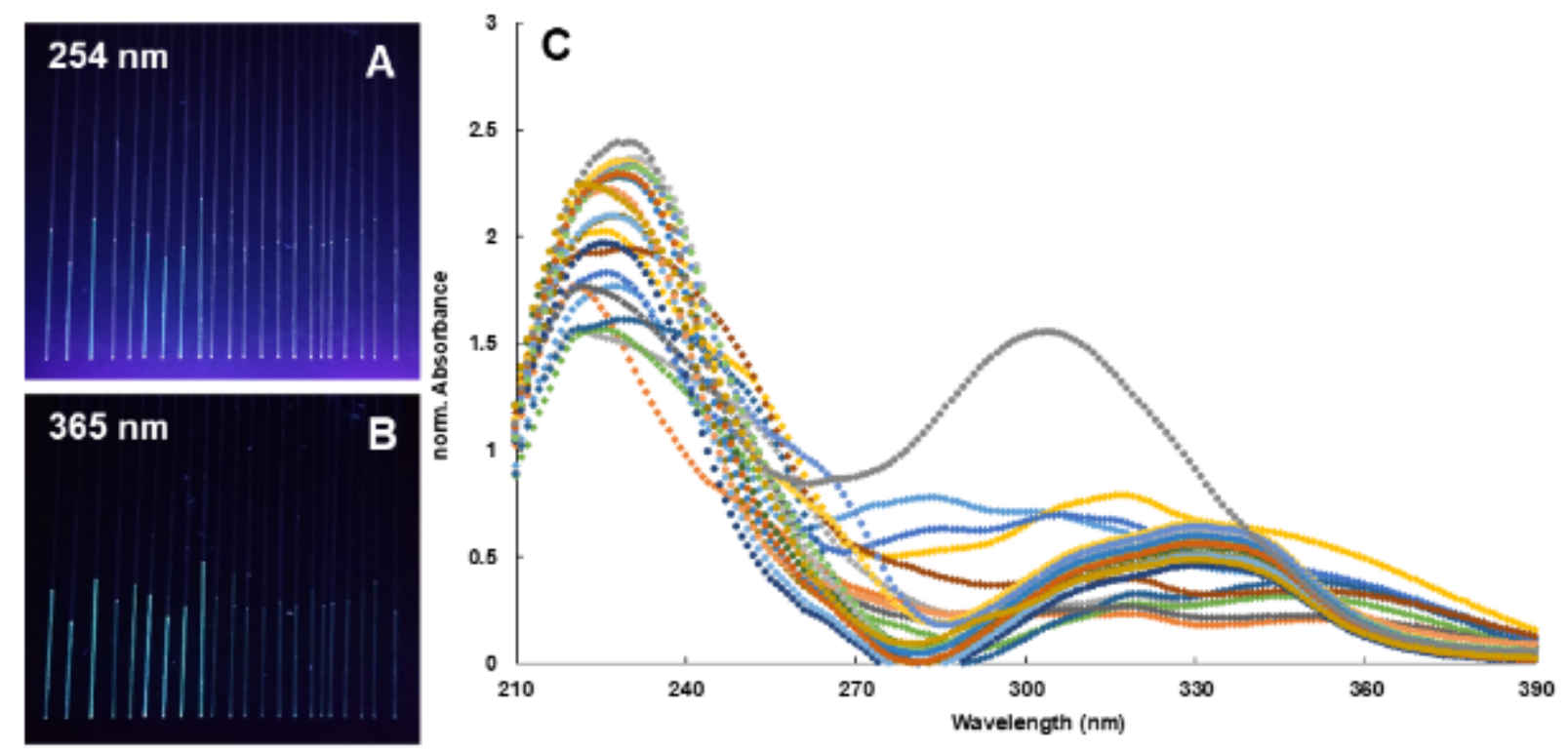

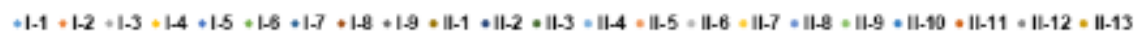
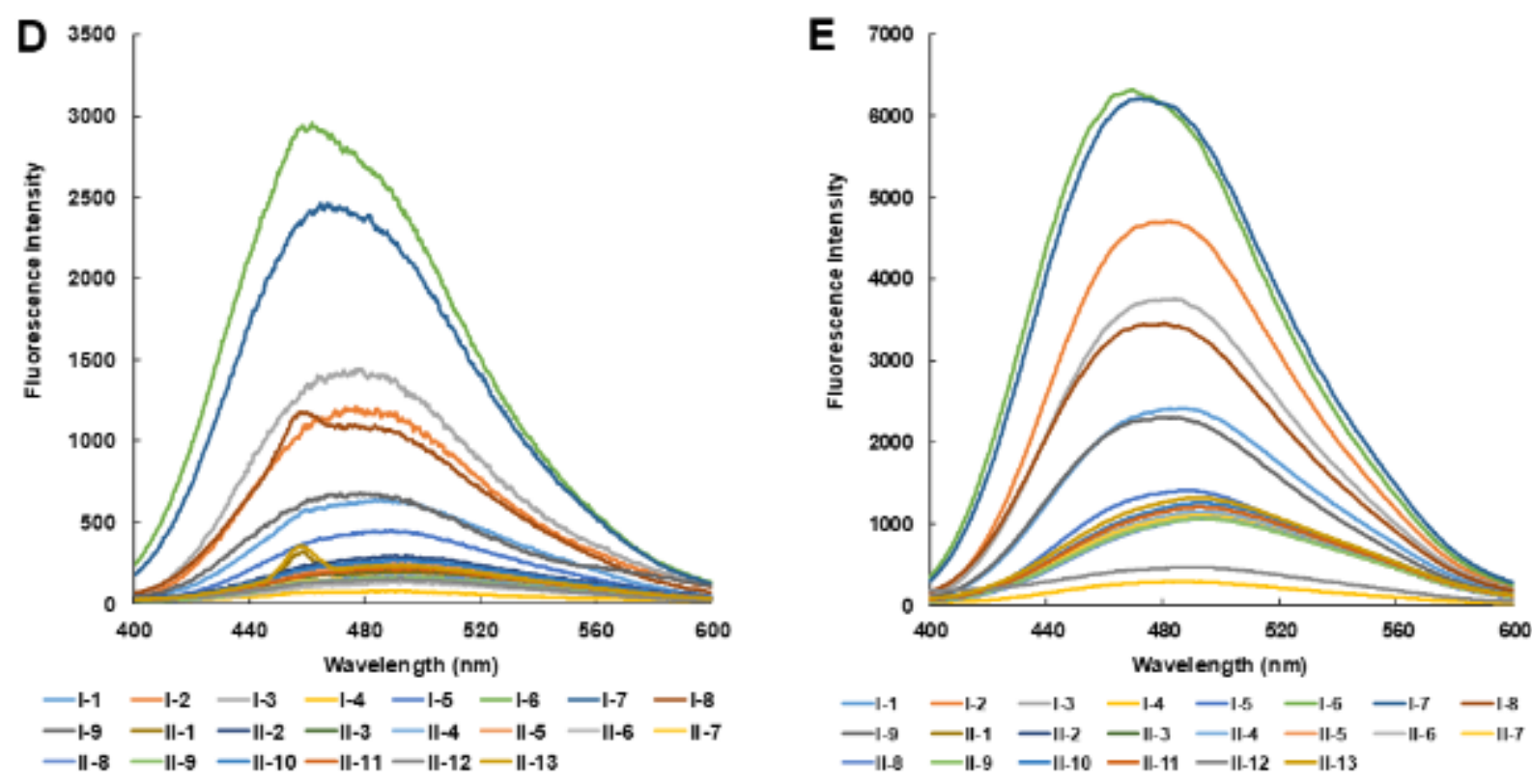

\section{Figure 4}

Methanol solutions $(50 \mu \mathrm{M})$ of the target compounds I-1 - I-9 and II-1 - II-13 under $254 \mathrm{~nm}(\mathrm{~A})$ and 365 $\mathrm{nm}$ (B) light irradiation, and UV-Vis absorption spectra (C), and fluorescence emission spectra at the first (D) and second (E) excitation wavelengths 


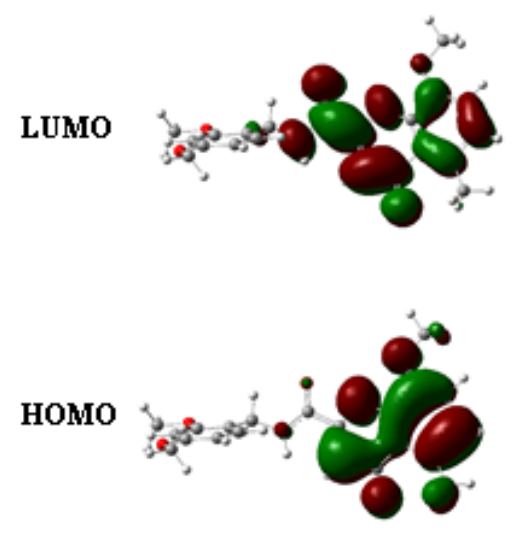

I-6
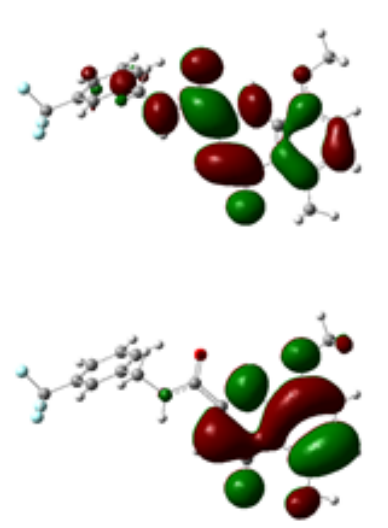

I- 7
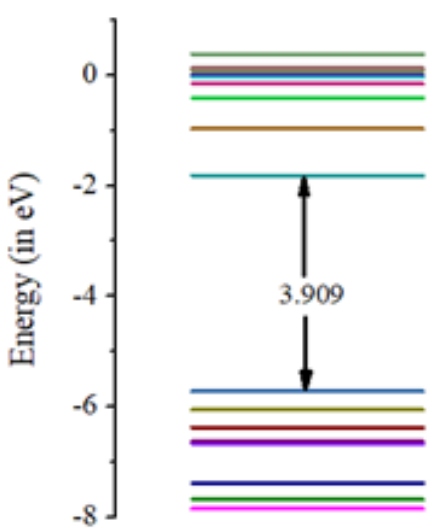

I-6

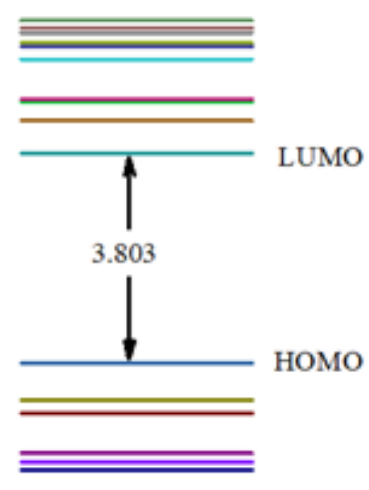

I-7

Figure 5

HOMO and LUMO orbitals of molecules I-6 and I-7.

\section{Supplementary Files}

This is a list of supplementary files associated with this preprint. Click to download.

- Scheme1.png

- supportinginformation.docx 